\title{
Effect of Mould Orientation on the Field-Dependent Properties of MR Elastomers under Shear Deformation
}

\author{
Muntaz Hana Ahmad Khairi ${ }^{1}{ }^{\mathbb{D}}$, Saiful Amri Mazlan 1,2,*, Ubaidillah 3,*D, Nur Azmah Nordin ${ }^{1}$, \\ Siti Aishah Abdul Aziz ${ }^{1}$ and Nurhazimah Nazmi ${ }^{1}$ D
}

1 Engineering Materials and Structures (eMast) iKohza, Malaysian-Japan International Institute of Technology, Universiti Teknologi Malaysia, Jalan Sultan Yahya Petra, Kuala Lumpur 54100, Malaysia; hana5700@gmail.com (M.H.A.K.); nurazmah.nordin@utm.my (N.A.N.); sitiaishah.aa@utm.my (S.A.A.A.); nurhazimah@utm.my (N.N.)

2 Advanced Vehicle System (AVS) Research Group, Universiti Teknologi Malaysia, Jalan Sultan Yahya Petra, Kuala Lumpur 54100, Malaysia

3 Mechanical Engineering Department, Faculty of Engineering, Universitas Sebelas Maret, Jl. Ir. Sutami 36A Kentingan Jebres, Surakarta 57126, Indonesia

* Correspondence: amri.kl@utm.my (S.A.M.); ubaidillah_ft@staff.uns.ac.id (U.)

Citation: Ahmad Khairi, M.H.; Mazlan, S.A.; Ubaidillah; Nordin N.A.; Aziz, S.A.A.; Nazmi, N. Effect of Mould Orientation on the Field-Dependent Properties of MR Elastomers under Shear Deformation. Polymers 2021, 13, 3273. https:// doi.org/10.3390/polym13193273

Academic Editor: Shazed Aziz

Received: 31 August 2021

Accepted: 22 September 2021

Published: 25 September 2021

Publisher's Note: MDPI stays neutra with regard to jurisdictional claims in published maps and institutional affiliations.

Copyright: (c) 2021 by the authors. Licensee MDPI, Basel, Switzerland. This article is an open access article distributed under the terms and conditions of the Creative Commons Attribution (CC BY) license (https:// creativecommons.org/licenses/by/ $4.0 /)$.

\begin{abstract}
In this study, magnetorheological elastomer (MRE) was fabricated using an electromagnetic device with a new configuration mold at the orientation of $0^{\circ}, 45^{\circ}$ and $90^{\circ}$. This new curing concept enhanced the alignment of carbonyl iron particles (CIPs) within the silicone matrix in the presence of silicone oil (SO) during solidifying, by eliminating air gaps to prevent magnetic flux losses. Using a mold made of steel, which is a magnetic material, the mold functions as a guide for concentrated magnetic flux of $0.315 \mathrm{~T}$ to pass through the MRE sample. Scanning electron microscopy (SEM) was used to observe the surface morphology of the fabricated MRE samples particularly the alignment of the CIPs. The field-dependent dynamic properties of the MREs were measured using a rheometer. The analysis implied that the effectiveness of the MRE operating under shear deformation with this curing concept provided the highest magneto-induced modulus of $1.01 \mathrm{MPa}$ when a $45^{\circ}$ orientation mold is used, with relative magnetorheological (MR) effect value up to $918 \%$, followed by $0^{\circ}$ mold orientation with $0.79 \mathrm{MPa}$ magneto-induced modulus and $646 \%$ relative MR effect. The high modulus properties offered by this MRE are believed to be potentially useful in industrial applications where a high range of stiffness is required particularly in the shear direction.
\end{abstract}

Keywords: magnetorheological elastomer; orientation; alignment; anisotropic; isotropic; silicone oil; plasticizer; mold; stiffness; $45^{\circ}$; modulus; magneto-induced; MR effect

\section{Introduction}

Magnetorheological elastomer (MRE) has attracted much interest as the material can exhibit a desirable performance owing to the stability of magnetizable particles in a solid-based matrix [1-4]. MRE is a polymer composite, which mainly consists of a rubber matrix, magnetic particles as a reinforcement phase, and optional additives for acquiring specific properties such as increased stability and providing much more superior bonding between the matrix and the magnetic particles [5-9]. Depending on the curing process, it can be classified into two categories; isotropic MRE, which contains randomly distributed particles, and anisotropic or aligned MRE, which contained polarized particles following the direction of the magnetic field during the fabrication process [10,11]. Due to this fact, anisotropic MRE possesses closer gaps between the magnetic particles, as compared to that in isotropic MRE. This can lead to strong interactions between the particles, which make it much more sensitive towards the change in the intensity of the magnetic field causing a change in the stiffness of the material. The changes in the stiffness, normally in the range between 0.1 to $0.6 \mathrm{MPa}$, are called MR effect and it becomes a crucial parameter to evaluate 
the performance of MRE [12-14]. Correlation between particles distribution and MR effect have been supported by the numerical results of Xia et al. [6], who demonstrated that the particles' distribution have significant effects on the MR effect. Moreover, according to Samal et al. [10], the sample dimension can also affect the distribution of CIPs in the MRE thus consequently affecting the MR effect.

Recently, MRE was developed to acquire a high MR effect with the addition of plasticizer [7,15-17] and by orienting the magnetic particles particularly carbonyl iron particles (CIPs) [18] or a combination of both approaches [19]. A plasticizer, typically a solvent, is added to a synthetic resin to produce or promote plasticity and flexibility of polymer materials. With this method, the MRE becomes softer and exhibits a higher relative MR effect, as a result of lower zero-field modulus or initial storage modulus [20,21]. Therefore, the interaction between magnetic particles and matrix phase during the fabrication process has been improved and at the same time, has modified the stiffness of MRE. Moreover, the plasticizer acts as a lubricant and allows molecular chains of rubber to glide easily during the curing process, thus decreasing the adhesiveness of a rubber matrix [22,23]. In other words, this advantage of plasticizer helps in aligning the process of the CIPs in an anisotropic MRE. As a result, the CIPs are aligned utterly following the direction of the magnetic field in the MRE.

The use of plasticizer was introduced by Lokander et al. [16], namely di-2ethylhexylphthalate into isotropic nitrile rubber-based MRE. This study obtained a slight improvement in the relative MR effect of MRE; however, no quantitative data were shown by them. Furthermore, Chen et al. [17] studied the influence of mineral oil as a plasticizer on the shear storage modulus of anisotropic natural-based MRE. In their study, the plasticizer used was between 10 to $20 \mathrm{wt} . \%$ and the result revealed that the addition of $10 \mathrm{wt} . \%$ mineral oil provided an initial storage modulus of $1.4 \mathrm{MPa}$, and magneto-induced modulus of MRE was $0.23 \mathrm{MPa}$. Meanwhile, when the amount of mineral oil increased to $20 \mathrm{wt} . \%$, the initial shear storage modulus and magneto-induced modulus of MRE became 0.9 and $0.7 \mathrm{MPa}$, respectively. In brief, the relative MR effects of MRE were at $21 \%$ and $78 \%$ when 10 and $20 \mathrm{wt} . \%$ mineral oil were added, respectively. For the case of silicone rubber-based MRE, Gong et al. [15] studied the effect of silicone oil (SO) in an anisotropic MRE. The addition of $15 \mathrm{wt} . \%$ SO exhibited a relative MR effect up to $400 \%$ with the range of storage modulus was between 0.25 to $1.25 \mathrm{MPa}$, at $1 \mathrm{~T}$. The anisotropic MRE contained $70 \mathrm{wt} . \%$ CIPs and was cured under the magnetic field of $1.5 \mathrm{~T}$. Therefore, the addition of plasticizer has proven to enhance the MR effect of the MRE.

Other than the use of plasticizer, the MR effect can be enhanced by orienting the CIPs in the MRE during the curing process; though the study is rather limited. Previously, the conventional anisotropic MRE had an orientation of $0^{\circ}$ of CIPs to the $y$-axis $[15,24]$. However, the study was evolved by focusing on the various orientations of CIPs in anisotropic MRE. A study on CIPs orientation other than $0^{\circ}$ anisotropic MRE was conducted by Boczkowska et al. [18]. Polyurethane-based MRE was developed with the same weight fraction of $60 \mathrm{wt} . \%$ magnetic particles with different particle orientations including $0^{\circ}$, $45^{\circ}$, and $90^{\circ}$. The experiment revealed that the largest MR effect was obtained at $45^{\circ}$ to the direction of the magnetic field. The order of the values of the MR effect versus the orientation was $90^{\circ}<0^{\circ}<45^{\circ}$. However, the study did not show how they obtained CIPs orientation of $0^{\circ}, 45^{\circ}$, and $90^{\circ}$ during the fabrication process; moreover, no microstructure images of the MRE with $0^{\circ}, 45^{\circ}$, and $90^{\circ}$ were disclosed.

Rather than individually focusing on the use of plasticizer and CIPs orientation, MR effect can also be improved by combining both approaches. Tian et al. [25] introduced SO plasticizer to the fabrication of anisotropic silicone rubber-based MRE with $45^{\circ}$ of CIPs orientation by rotating the non-magnetic mold to $45^{\circ}$ relative to the applied magnetic field that is in the vertical direction. The team found that the MR effect in one shear direction was at $580 \%$ compared to that in the opposite direction, which was at $480 \%$. In that study, $15 \mathrm{wt} . \% \mathrm{SO}$ was used as a plasticizer with $70 \mathrm{wt} . \%$ of CIPs as magnetic particles in the fabricated MRE. In another study, Yao et al. [19] developed a new model to describe the 
shear behavior of MRE with particle chain's orientation including $0^{\circ}, 45^{\circ}$, and $90^{\circ}$. The model portrayed micro-mechanical interaction between particles in the MRE, based on the theory of magnetic dipoles and magnetic field coupling between particles that aligned in the chain. The results of the model revealed that the MR effect of MRE has a strong dependence on the orientation of the particle's chains and $45^{\circ}$ of CIPs orientation resulted in the highest MR effect [19]. However, in experimental verification, the selection of a low percentage of CIPs and SO, particularly $33 \mathrm{wt} . \%$ and $0.4 \mathrm{wt} . \%$, respectively, resulted in a low relative MR effect of MRE, which was $115 \%$. The low MR effect of MRE showed that the composition of CIPs and SO as plasticizer was crucial and must be vividly considered to fabricate MRE with the expectation of a higher MR effect. This is despite the fact that anisotropic MRE was developed to acquire a high MR effect with the addition of plasticizer [15] and in various CIPs orientations [18] or a combination of both approaches [19,25]. Current developed anisotropic MREs have MR effect below 600\%, whereas if the MR effect can be achieved at more than $600 \%$, it can be beneficial to applications that require a high MR effect, especially in the shear direction. The obtained MR effect which is below $600 \%$ of MRE was probably due to the limitation of the curing device, which led to the flux losses during the curing process, hence affecting the CIP's alignment.

Generally, the concept of a curing device that other researchers used in the past for anisotropic curing mostly was made from a non-magnetic materials slab's mold that was divided into two parts, top and bottom $[2,19,25,26]$. The sample was placed in the middle, sandwiched between the upper and bottom mold. In this design, the non-magnetic mold has not properly guided the magnetic flux to flow into the MRE. Moreover, this design might create high magnetic flux losses due to the air gap between the mold and the coil, resulting in insufficient magnetic flux that penetrated the MRE sample during the curing process, consequently, affecting the alignment process of CIPs. As stated by Tian et al. [25], the measurements of flux density were taken outside of the non-magnetic mold and it was found that the magnetic flux density was more than $0.3 \mathrm{~T}$ to align the particles particularly at $45^{\circ}$ within the matrix phase. However, it is unsure that the same $0.3 \mathrm{~T}$ was flowing inside the mold to align the magnetic particles in the MRE, due to their curing device design; the magnetic flux might be lost to the air. Hence, the alignment process of CIPs in an MRE at a specific degree might have deteriorated that subsequently affecting the corresponding MR effect as well.

On the other hand, another anisotropic curing setup was presented by Samal et al. [27], where the MRE sample was designed cylindrically located on the top of the curing device. The simulation of magnetic distribution was reported to be different at the top, middle and bottom of the MRE sample, and the average value of magnetic induction concluded to be around $0.3 \mathrm{~T}$ after considering the ranges from the top level to the bottom of the MRE sample. The mentioned studies used plasticizer and orientation of CIPs in an MRE could result in improvement of the MR effect. However, until now the maximum modulus increase for the MRE has been reported to be up to $0.6 \mathrm{MPa}$ with magnetic particles at about $70 \mathrm{wt} . \%$ [27] probably due to the poorly aligned CIPs inside the MRE caused by the insufficient magnetic flux penetrating the MRE during the curing process. Thus, it is an opportunity to provide a uniform magnetic flux to ensure the particles are aligned (assemble into chain-like microstructures) before being locked up in the rubber-based matrix as a cured MRE in a solid-state [1].

The main technical contribution of this work is to obtain MRE (employed with SO) with a high MR effect of more than $0.60 \mathrm{MPa}$ magneto-induced modulus for widely industrial application. To achieve that, the magnetic response between the CIPs must be improved by enhancing the alignment of CIPs. This can be done by modifying the existing anisotropic curing device by using a "new configuration magnetic flux mold" that consists of a cylindrical mold which is inserted into the magnetic coil without an air gap to prevent magnetic flux loss, and the molds that are made from a magnetic material, which functions as guidance for the concentrated magnetic flux flow in the MRE mold. MRE sample (1 mm thickness) was designed to be sandwiched between 
the top and bottom of mold for enhanced alignment and uniform magnetic distribution when the concentrated magnetic flux was passing through the MRE during the curing process. The molds were employed with $0^{\circ}, 45^{\circ}$, and $90^{\circ}$ orientations (to the $y$-axis) to investigate the effect of different orientations towards the MR effect. After fabricating the proposed MRE samples, observations via scanning electron microscopy (SEM) are made to observe the particles' alignments in the samples. Subsequently, the field-dependent dynamic viscoelastic properties of the MREs were measured using a rheometer. The measured results were compared, and the effectiveness of the proposed MRE was validated to determine the enhancement of the magneto-induced and relative MR effects. It should be noted here that to the best of the authors' knowledge, there is no MRE using this magnetic flux mold's concept for orientation specifically at $0^{\circ}, 45^{\circ}$, and $90^{\circ}$ so far.

\section{Materials, Fabrications, and Methods}

\subsection{Materials}

The elastomer used as a matrix material for the manufacture of MREs is silicon rubber base, type RTV-two NS625 supplied by Nippon Steel. It is physically a white flowable liquid that would have a hardness of 22-50 $\AA$ after the completed curing process. The magnetic particles, CIPs are from BASF, Germany with an average size of $6 \mu \mathrm{m}$. The anisotropic curing condition using the new concept of mold where the particles are configured at $0^{\circ}$, $45^{\circ}$, and $90^{\circ}$ orientations as well as isotropically. Fabricated MRE samples were using $70 \mathrm{wt} . \%$ of CIPs with $15 \mathrm{wt} . \%$ of SO, followed by $15 \mathrm{wt} . \%$ of silicone rubber. SO plays an important role to soften the matrix which can reduce the zero-field storage modulus of MRE and enhanced the maximum stiffness change of MRE. Moreover, SO should improve the adhesive properties of the particles on the matrix surface [27]. It should be noted that one sample without $\mathrm{SO}$ was prepared for comparison, and viscosity test for uncured sample will be carried out to determine the MRE viscosity before the solidification process.

The required amount of silicone rubber, $\mathrm{SO}$, and CIPs were weighed accordingly. The materials were mixed thoroughly and uniformly using a mechanical stirrer for $20 \mathrm{~min}$. Then, the curing agent was added and the mixture was stirred again for $2 \mathrm{~min}$. This is to allow the curing process to occur and transform the paste-like sample to become solid. Next, the final mixture was poured into a customized steel mold $\left(0^{\circ}, 45^{\circ}\right.$, and $90^{\circ}$ molds) with the size of $1 \mathrm{~mm}$ in thickness and $70 \mathrm{~mm}$ diameter and left for solidification with the absence of magnetic field, particularly for the isotropic condition. For the $0^{\circ}, 45^{\circ}$, and $90^{\circ}$ orientations mold, the mixture was cured with the presence of a magnetic field in terms of current applied from electromagnetic circuit. For the isotropic condition, the mold of $0^{\circ}$ was used but without a magnetic field applied during the respective curing process so that the magnetic particles were homogeneously distributed in the MRE. All MRE samples are labelled as MRE $0^{\circ}, \operatorname{MRE} 45^{\circ}, \operatorname{MRE} 90^{\circ}$ and isotropic MRE.

\subsection{Curing Device and Simulation of Magnetic Flux}

The curing device was used with a new concept of mold configuration to generate a focused-guided magnetic field with the electromagnetic circuit in the sample during the curing process of the anisotropic MRE with different mold orientations. In general, the highest magnetic field strength with a certain alignment of magnetic fluxes that passed through the sample is the foremost requirement to form anisotropic MRE. In other words, the new configuration of electromagnetic circuit and mold can generate the maximum magnetic field strength into the sample and at the same, the magnetic field can be controlled via the electromagnetic circuit. This new curing device was comprised of coil, casing, separator and mold. The coil, 18 AWG with a diameter of $1.11 \mathrm{~mm}$, was made of copper and was rolled up to the non-magnetic bobbin (polyethylene) with 2270 turns. The casing and the mold are made of mild steel, while the separators, which are used for directing the magnetic field into the mold, are made of polyethylene for mold $90^{\circ}$ and aluminum for mold $45^{\circ}$. The schematic diagram of the modified curing device is illustrated in Figure 1 and details of the input parameter of the components are listed in Table 1. 


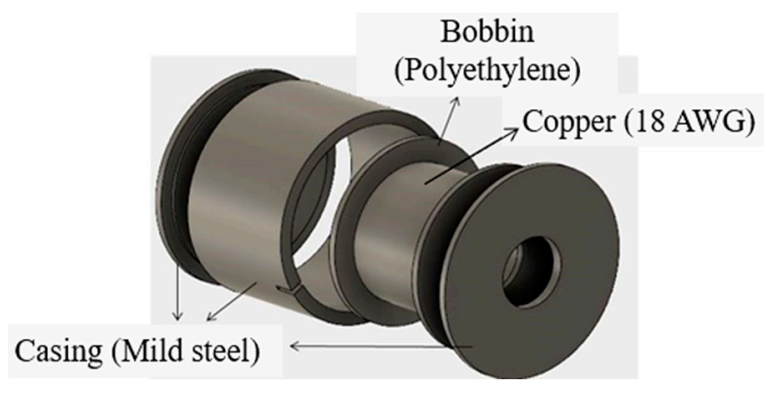

(a)

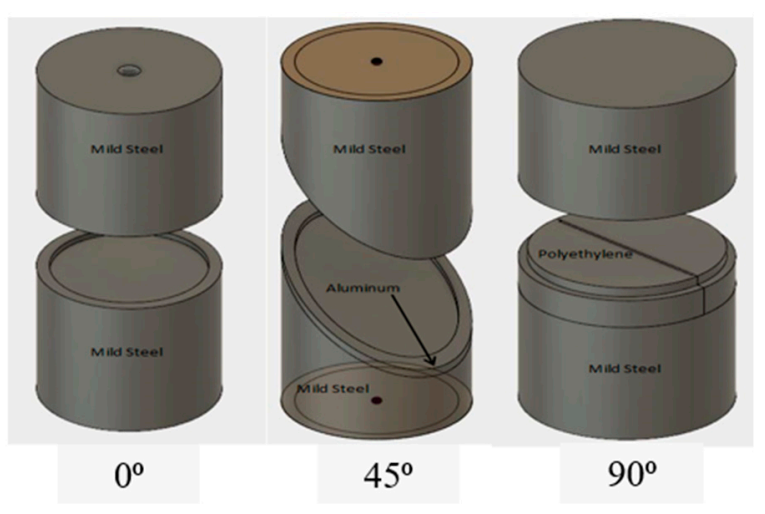

(b)

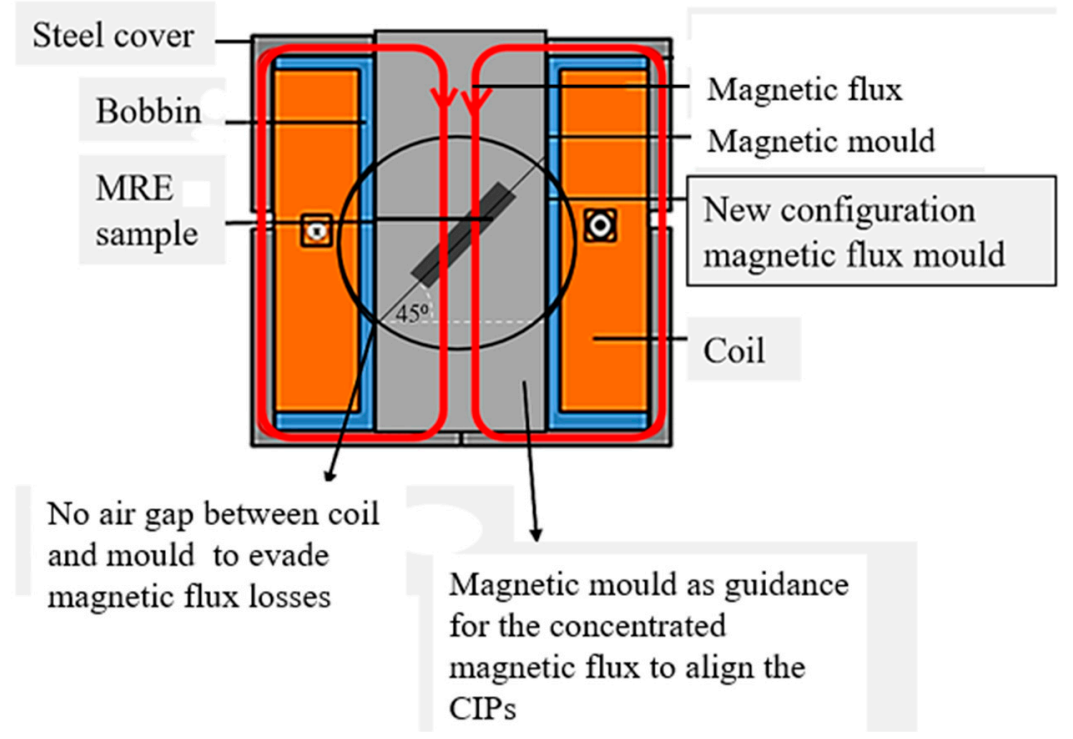

(c)

Figure 1. The anisotropic curing device (a) design of casing (b) design of mold (c) schematic diagram.

Table 1. Input parameter of the components in the curing device of anisotropic MRE to be run in Finite element method magnetic (FEMM).

\begin{tabular}{cc}
\hline Component Name & Material \\
\hline Mould $\left(0^{\circ}, 45^{\circ}\right.$ and $\left.90^{\circ}\right)$ & Mild steel \\
\hline Steel Cover & Mild steel \\
\hline Electromagnetic Coil & Copper (18 AWG $(2270$ turns $))$ \\
\hline Casing & Mild Steel \\
\hline Bobbin & Mild Steel \\
\hline Separator for $45^{\circ}$ and $90^{\circ}$ mold & Non-magnetic \\
\hline MRE sample & Magnetic and \\
\hline
\end{tabular}

Finite element method magnetics (FEMM) was performed during designing the mold concept to measure the magnetic flux density that passes through the sample as illustrated in Figure 2 based on input parameters in Table 1. It can be observed that the distribution of magnetic flux density, shown in red (darkest shade), signifies the presence of maximum magnetic flux in that region. The green-yellow (lighter shade) color signifies the presence 
of moderate magnetic flux. The minimum magnetic flux region is denoted by the blue color (white or no shade) especially at the casing and surrounding region. The simulation was performed to obtain at least $0.3 \mathrm{~T}$ of magnetic flux density to align the particles within the matrix phase as stipulated by Tian et al. [25] and Samal et al. [27]. As a result of the simulation, $0.315 \mathrm{~T}$ of magnetic flux density, as shown in Figure $2 \mathrm{~b}$, was achieved at applied current of $0.2 \mathrm{~A}$. The current was adjusted accordingly to maintain the same value of magnetic flux density for $45^{\circ}$ and $90^{\circ}$ molds. The actual measurement of the magnetic flux density at the fabricated molds was carried out via a specific hole on the casing wall, using a tesla meter.
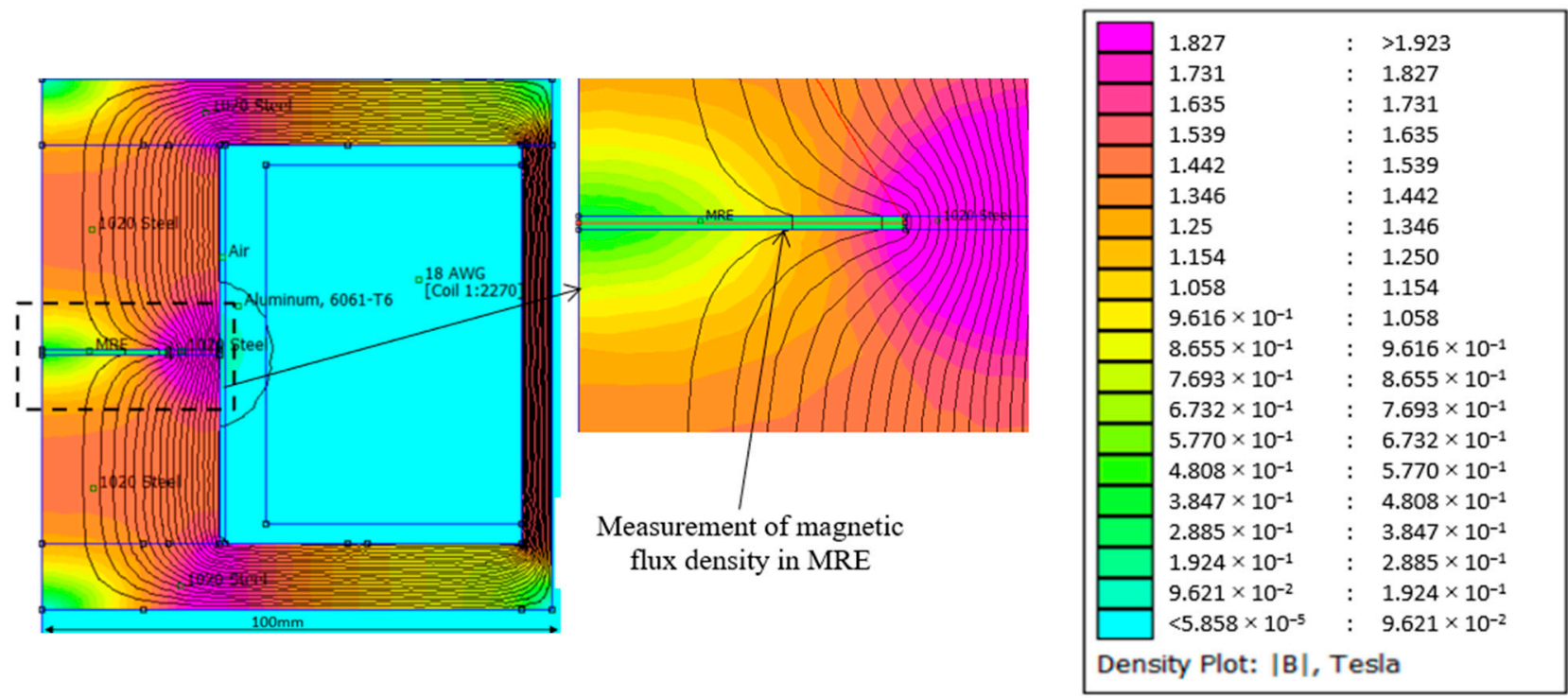

(a)

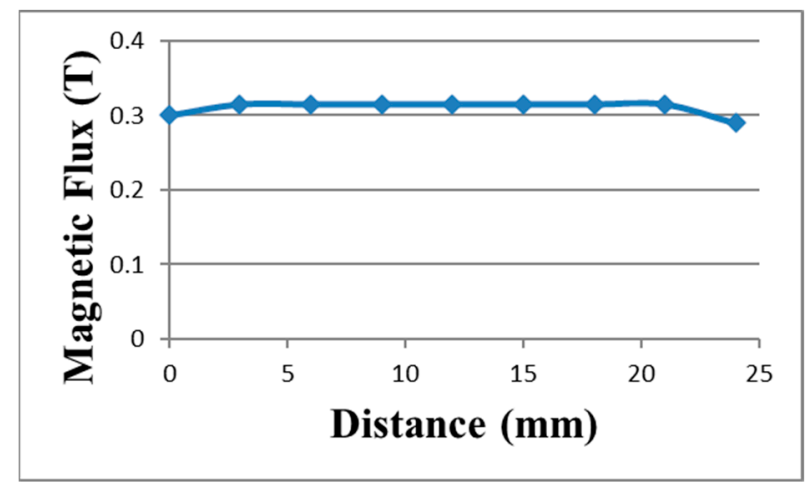

(b)

Figure 2. Simulation of magnetic flux density using FEMM: (a) distribution and (b) the value of magnetic flux across the MRE sample.

As for the comparison, the concept of mold that other researchers used in the past for anisotropic curing was reproduced through simulation as shown in Figure $3[2,19,25,26]$. As observed through FEMM, the mold yielded magnetic flux density of $0.1 \mathrm{~T}$ as opposed to the new configuration, where it achieved $0.315 \mathrm{~T}$ when same setup (design, number of coil turns, and current input) was applied. Moreover, the values of magnetic flux density were uneven across the MRE that deteriorated alignment of CIPs inside the MRE. 


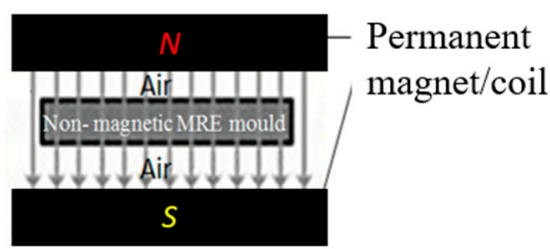

(a)

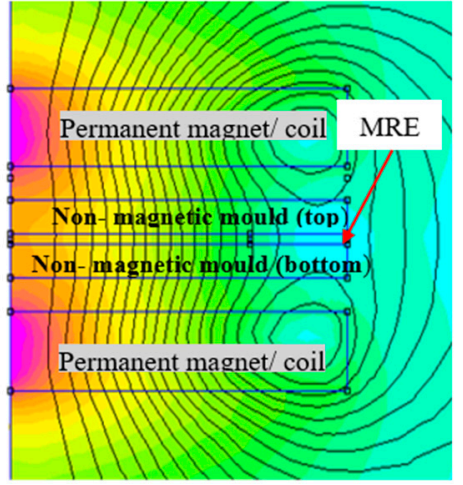

(b)

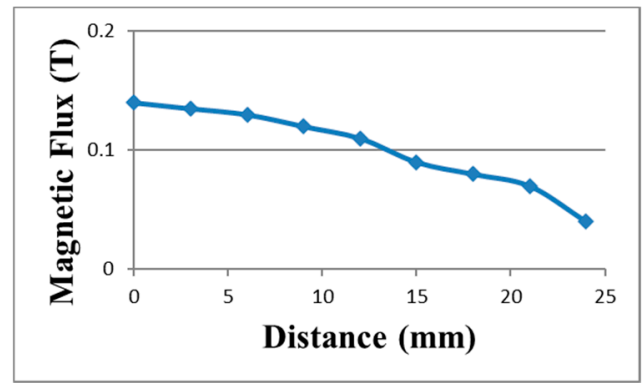

(c)

Figure 3. Simulation of magnetic flux density using FEMM of commonly used in previous studies: (a) Schematic drawing of the curing setup (b) distribution (c) value of magnetic flux across the MRE sample.

Figure 4 shows the experimental set-up used for curing process of the anisotropic MRE with $45^{\circ}$ mold that cured under the presence of magnetic flux density $(0.315 \mathrm{~T})$. The same coil was used to fabricate $\mathrm{MRE} 0^{\circ}, 45^{\circ}$, and $90^{\circ}$ molds by placing them individually at the center of the electromagnetic coil. The location of the flux density measurement at a specific space inside the chamber was also disclosed.

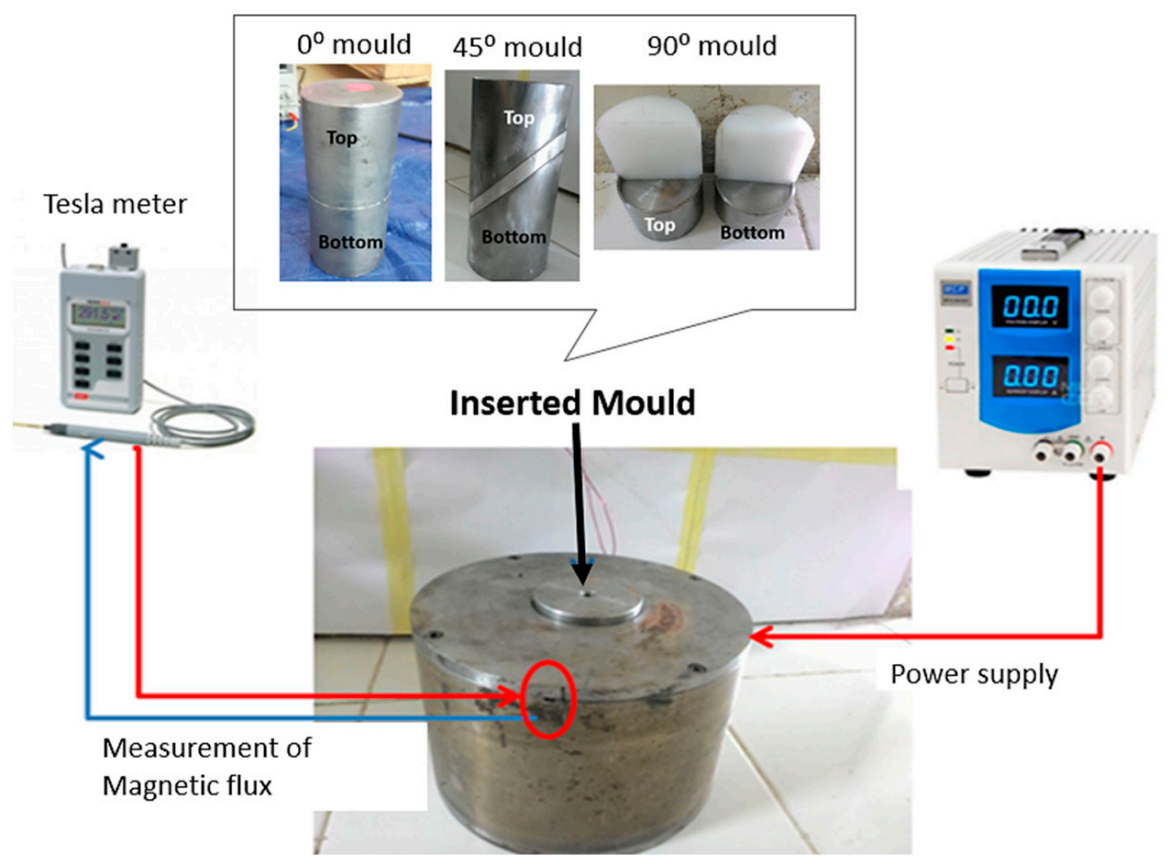

Figure 4. The setup of a curing device for anisotropic MRE with various mold orientations was inserted.

Meanwhile, Figure 5 shows an illustration of the alignment of CIPs in the matrix phase during the solidification process in the molds of $0^{\circ}, 45^{\circ}$ and $90^{\circ}$ to the $y$-axis. The mold was used as guidance for the concentrated magnetic flux to flow parallelly from top to bottom parts of the mold. The high magnetic flux that passed through the MRE sample, and sandwiched between the mold's parts, resulted in the enhancement of the alignment of CIPs within the silicone matrix phase. On the other hand, another anisotropic curing setup was introduced by Samal et al. [27], where the MRE samples were designed cylindrically located on the top of the curing device with length of $20 \mathrm{~mm}$ and a radius of $5 \mathrm{~mm}$ in contact, by its lower and upper surface, with copper slabs in the circuit of the magnetic 
field. The magnetic distribution was reported to be different at the top, middle and bottom of the MRE sample. In this work, as mentioned in the previous text, the MRE sample $(1 \mathrm{~mm}$ thickness) to be sandwiched between the cylindrical magnetic mold that is inserted into the electromagnetic coil for better alignment and uniform magnetic distribution throughout the MRE sample. The thickness of the sample was designed to be $1 \mathrm{~mm}$, suitable for rheology test using a rheometer.

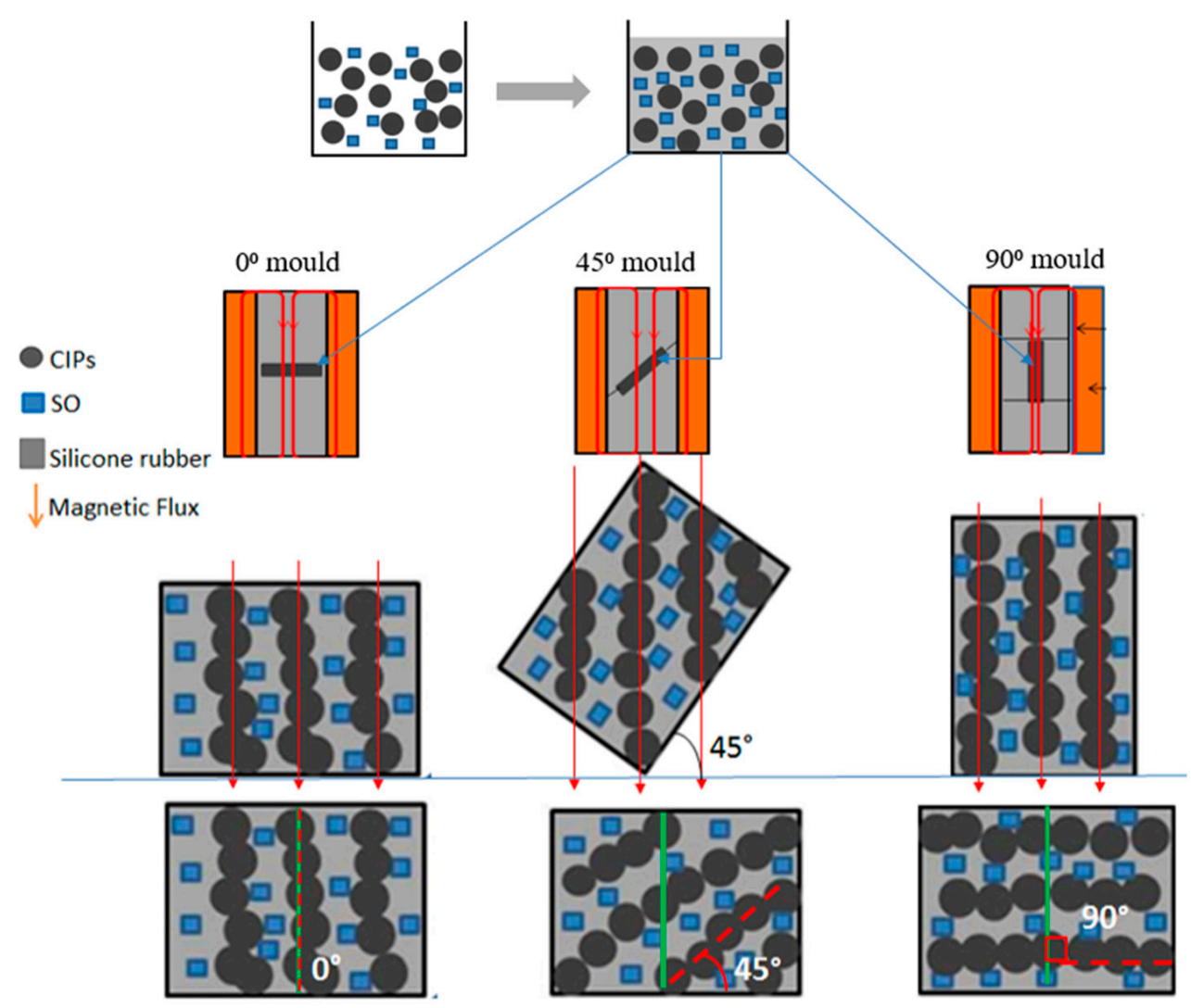

Figure 5. Illustration of CIPs orientation with $0^{\circ}, 45^{\circ}$ and $90^{\circ}$ to the $y$-axis during curing process.

\subsection{Magnetic Properties Measurement and Morphological Observation}

The magnetization curves were measured at room temperature using Vibrating Sample Magnetometer (Micro Sense, EZ series). The working principle of using VSM was started by placing the MRE sample at a constant magnetic field. The MRE sample was protected by wrapping it with a polytetrafluoroethylene (PTFE). A constant magnetic field would magnetize the sample, and the magnetic domains of the particles vibrated and tended to align, along the direction of the magnetic field. The magnetic dipole moments of the sample created a magnetic field around the sample, and the changes of the magnetic fields were sensed by a coil. The magnetization of the sample depended on the strength of the constant magnetic field. The magnetic field was ranging from -1250 to $1250 \mathrm{kA} / \mathrm{m}$ and the graph of magnetization $(\mathrm{M})$ versus magnetic field strength $(\mathrm{H})$ was then generated. The important outputs generated from the tests including magnetic saturation (Ms), coercivity (Mc) and retentivity (Mr), which determined the magnetic behavior of the MRE materials.

The morphological characterization of the MRE samples was observed under Low Vacuum Scanning Electron Microscopy (LVSEM, JEOL JSM-IT300, Tokyo, Japan) at an acceleration voltage of $15 \mathrm{kV}$ up to 1000 times magnifications. The LVSEM is used to scan the cross-section surface of samples to create an image using focused beam electrons. The MRE samples were coated with a gold layer on the surface to avoid any charging during observation. Then, a sample was mounted rigidly to a specimen holder using a conductive adhesive and it was placed in the sample chamber. The emitted electron beam scanned the cross-section of the samples, where the electrons in the beam interact with the 
sample, producing various signals that can be used to obtain information about the surface topography and composition. The determination of the elements in MRE were confirmed through energy-dispersive X-ray fluorescence (EDX, JEOL, JED-2300, Tokyo, Japan).

\subsection{Rheological Properties Test}

In the rheology test, all samples were examined in uncured and cured conditions which were related to viscosity and viscoelastic investigation, respectively. The viscosities of the uncured MREs with and without $\mathrm{SO}$ were measured using a rotational rheometer (Physica MCR 302 from Anton Paar, Graz, Austria). The samples were tested at $25{ }^{\circ} \mathrm{C}$ controlled by the Viscotherm VT2 (Anton Paar, Graz, Austria) at a shear rate of $0.001-10 \%$, and the gap between the oscillatory disk was fixed at $1 \mathrm{~mm}$. The dynamic viscoelastic properties of the cured MRE samples were evaluated using the oscillatory rheometer MCR 302. The rheometer MCR302 was equipped with the current controller MRD70 to generate the required magnetic field through the system. The measurements were performed by sandwiching the MRE samples between a rotary disk and a parallel base plate, PP20 (Anton Paar, Graz, Austria). The dimensions of the samples were $20 \mathrm{~mm}$ in diameter and $1 \mathrm{~mm}$ thick. The samples were tested under different flux densities from 0 to $800 \mathrm{mT}$ with different frequencies at 1,5 and $10 \mathrm{~Hz}$. For the effect of strain amplitude sweep, the strains were fixed at $0.01,0.1$, and $1 \%$.

\section{Results and Discussions}

\subsection{Magnetic Properties}

The magnetization versus magnetic field (known as the hysteresis loop) was carried out to investigate the effect of magnetic characteristics of MRE with various CIPs orientations. Figure 6 shows the magnetic field strength $(\mathrm{kA} / \mathrm{m})$ versus magnetization of MRE samples with various $\mathrm{SO}$ concentrations in the form of hysteresis loops. In general, a magnetic moment (or magnetic dipole moment) is a measurement of a specific object to interact with the applied magnetic field. The object will tend to be arranged so that its magnetic moment vector aligns with the lines of the magnetic field. The hysteresis curve is obtained by increasing the magnetic field in both negative and positive directions. The magnetization will reach its saturation, known as magnetic saturation (Ms) with a further increase in the applied magnetic field, $\mathrm{H}$.

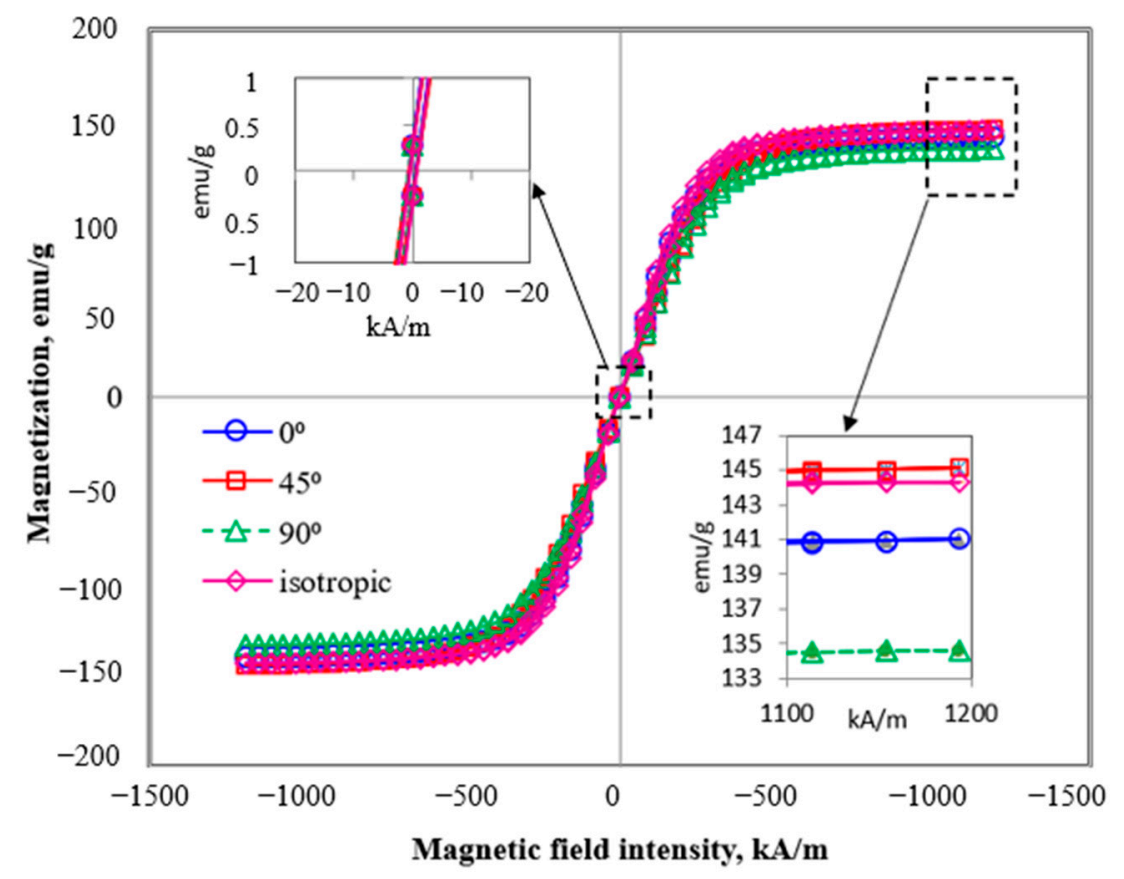

Figure 6. Hysteresis loops of MRE samples at $0^{\circ}, 45^{\circ}$, and $90^{\circ}$ orientations of mold, and isotropic MRE. 
Figure 6 illustrates the magnetization curves of MRE samples with various orientations. It is noted that those MRE samples have contained $15 \mathrm{wt}$ \% SO. Generally, the MRE samples with various orientations of CIPs exhibited narrow hysteresis loops indicating the soft magnetic characteristics of MRE since CIPs were used as reinforced magnetic particles in the rubber matrix. As observed in Figure 4, the magnetic hysteresis loop of MRE samples possesses a similar trend either at $0^{\circ}, 45^{\circ}$, or $90^{\circ}$ mold orientations and the $M_{S}$ values were noted to be in the range of $135-145 \mathrm{emu} / \mathrm{g}$. A small variation in $M_{\mathcal{S}}$ values $( \pm 10 \mathrm{emu} / \mathrm{g})$ might be due to the selected area of the sample for the magnetic properties test that consisted of various sizes of CIPs in the range of $1-10 \mu \mathrm{m}$. Thus, it is uncontrollable to acquire testing samples with the same distribution of CIPs. In addition, the retentivity, $M_{r}$ and coercivity, $H_{c}$ values of the MRE samples were not influenced by the variations of CIPs oriented as shown in Table 2. The $H_{c}$ and $M_{r}$ values were in the range of 8-8.3 and 0.27-0.29, with the deviation values were just around $\Delta 0.3$ and $\Delta 0.02$, respectively. $M_{r}$ stands for magnetic remanence of the magnetic material, which refers to the amount of magnetization that remained in the MRE after the applied magnetic field was removed. In the meantime, the $H_{c}$ is a measurement of the magnetizing force required to drive reverse magnetization once it has been saturated. These data show that the MRE with mold orientations at $0^{\circ}, 45^{\circ}$ and $90^{\circ}$ to the $y$-axis and isotropic did not affect the magnetization behavior of the MRE. On the other hand, the value of $M_{S}$ was dominantly dependent on the content of the CIPs within the MRE sample [28].

Table 2. Magnetic parameters from the magnetization curves.

\begin{tabular}{cccc}
\hline MRE Orientation $\left({ }^{\circ}\right)$ & $\boldsymbol{H}_{\boldsymbol{c}}[\mathbf{O e}]$ & $\boldsymbol{M}_{\boldsymbol{s}}[\mathbf{e m u} / \mathbf{g}]$ & $\boldsymbol{M}_{\boldsymbol{r}}[\mathbf{e m u} \mathbf{g}]$ \\
\hline 0 & 8.00 & 141 & 0.27 \\
\hline 45 & 8.30 & 145 & 0.29 \\
\hline 90 & 7.52 & 135 & 0.26 \\
\hline isotropic & 7.32 & 144 & 0.28 \\
\hline
\end{tabular}

\subsection{Morphology of the MRE}

The images of the cross-sectioned morphology of MRE samples are presented in Figure 7. It can be observed that for the isotropic MRE, the CIPs are dispersed randomly in the matrix phase, as shown in Figure 7a. Meanwhile, Figure 7b,c shows the alignments or chain-like structure of the anisotropic MRE with magnification of 500 and 1000 times, respectively. The CIPs are observed to be aligned forming the chain-like structure of particles, as a result of following the direction of magnetic field during curing process. According to Samal et al. [27], particles become magnetized and assembled into chain-like structures in a uniform magnetic field, due to magnetic dipole-dipole interactions, thus tend to align with the direction of external field. In this work, the new configuration magnetic flux mold concept generated a focused magnetic field into the mold and towards the pre-polymer MRE during the curing process. The interaction and attraction between the CIPs led them to align with the flow of magnetic field. As the curing reaction took place, the CIPs' chain-like structure became fixed and locked in the aligned manner in the matrix. As mentioned in the previous sub-section, the FEMM simulation shows that the concentrated and uniform magnetic flux of $0.315 \mathrm{~T}$ passed throughout the MRE sample, thus enhancing the alignment of the CIPs, consequently enhancing the MR effect that will be discussed in the rheology part in the next sub-section.

On the other hand, the distribution of the distance between adjacent particles to the resultant rheological properties of MRE was proposed by Suo et al. [29] using a magnetic dipole model based on chi-squared distribution. The mathematical models have considered the distance between magnetic particles and their magnetic dipole have affecting the rheological behaviour of the MRE. In another study, the correlation between the orientation of particles to the rheological properties of MRE was reported by Davis [30], whose study employed the magnetic dipole theory to compare the rheological behaviour of MRE before 
and after the dispersion of the magnetic particles. The study discovered that the rheological properties of the material were enhanced after the homogeneous dispersion of the magnetic particles was achieved. Additionally, the 3D images internal structure of the MRE were also investigated, and the simulation result revealed that the distribution of filler particles within the volume, in both isotropic and anisotropic distribution have a significant effect on the properties of MRE [31].

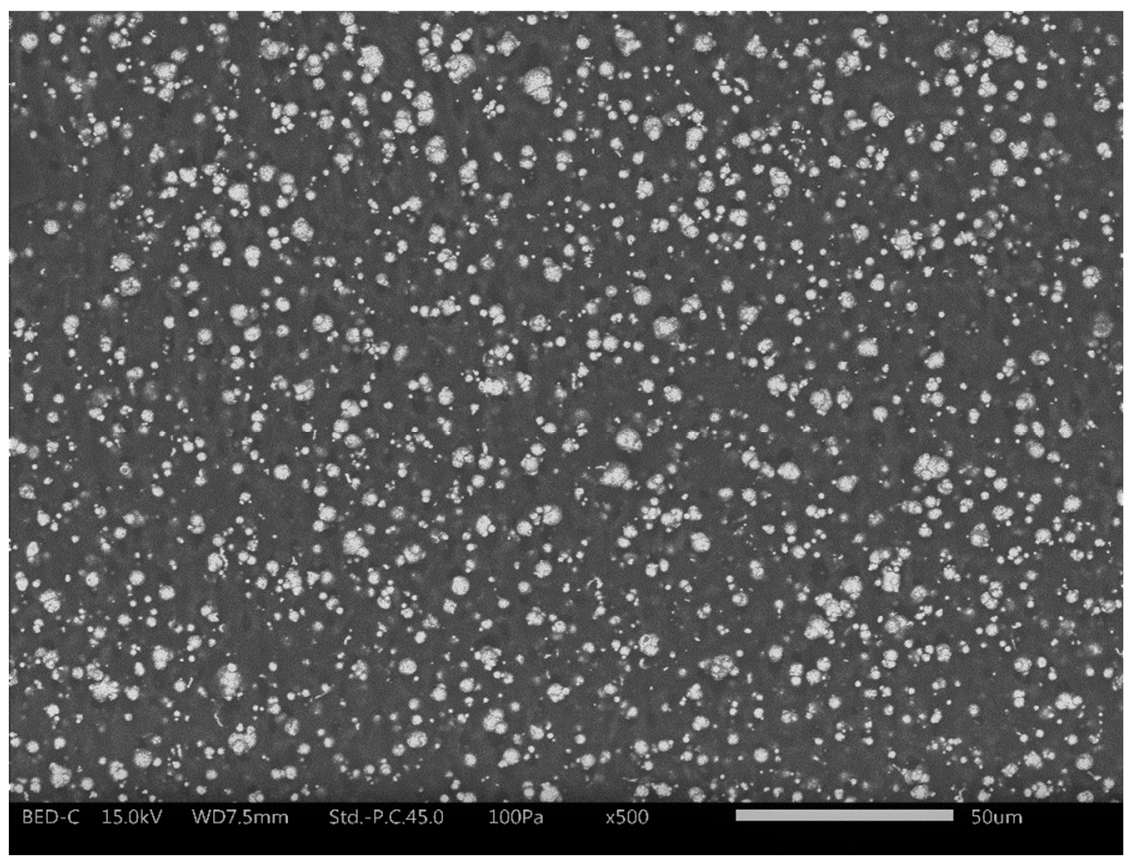

(a)

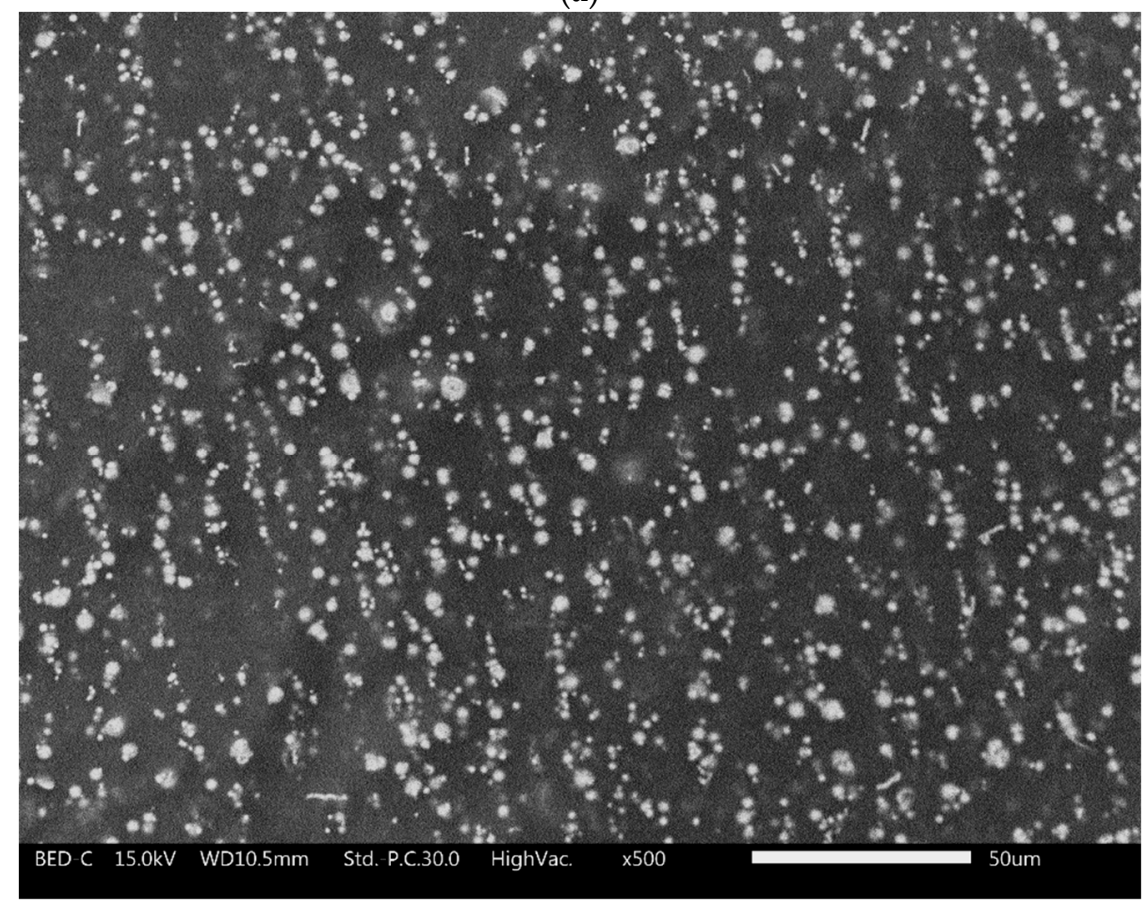

(b)

Figure 7. Cont. 


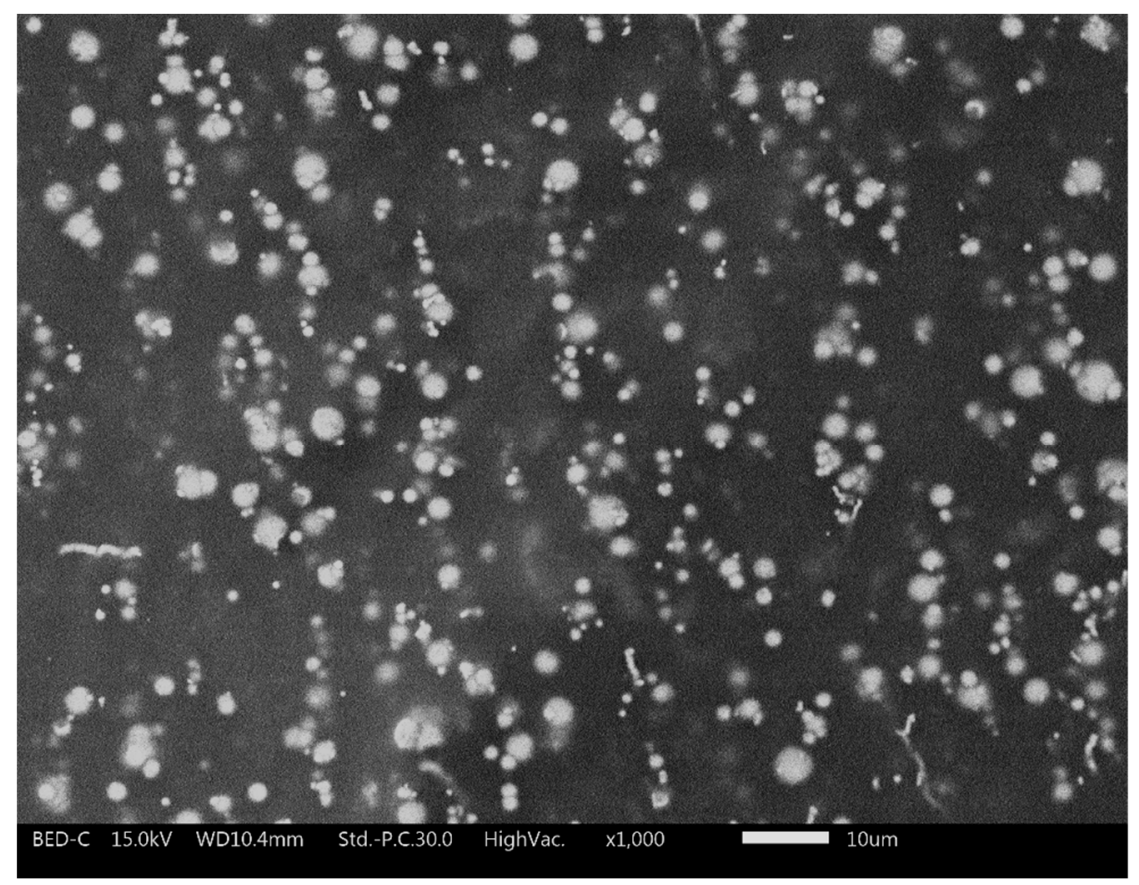

(c)

Figure 7. SEM images of MRE (a) isotropic at 500× magnifications (b) anisotropic at 500× magnifications (c) anisotropic at $1000 \times$ magnifications.

On the other hand, the elemental composition of MRE was identified through Energy Dispersive X-ray Analysis (EDX) mapping analysis as shown in Figure 8. The EDX mapping images traced the elements of $\mathrm{Si}, \mathrm{O}$, and $\mathrm{C}$ in both silicone rubber and SO. Meanwhile, the element Fe represented the CIPs in the MRE. Therefore, the mapping results were confirmed with the LV-SEM microstructure observations and MRE formulation.
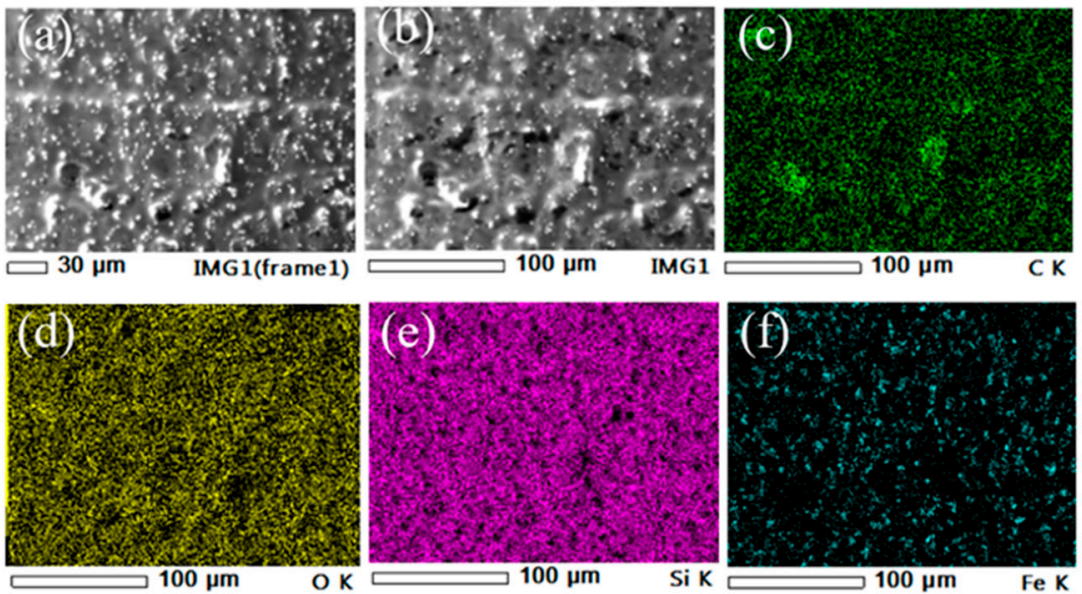

Figure 8. EDX mapping analysis of MRE sample consisting of $(\mathbf{a}, \mathbf{b})$ EDX region, (c) Carbon, green (C), (d) Oxygen, yellow (O), (e) Silicone, purple (Si) and (f) Iron, blue (Fe) elements.

\subsection{Rheological Properties: Effect of Mold Orientation}

The effects of mold orientations of $0^{\circ}, 45^{\circ}$, and $90^{\circ}$ on the storage modulus and relative MR effect of MRE under different magnetic fields are shown in Figure 9. The initial storage modulus, $\mathrm{G}_{0}$ and MR effect of MRE samples including isotropic and all orientations are summarized in Table 3. From the table, it is observed that the $\mathrm{G}_{0}$ of the MRE samples is not much influenced by the orientation of mold whether those are in $0^{\circ}, 45^{\circ}$ or $90^{\circ}$ to the $y$-axis, and also in isotropic conditions. Indeed, the fabrication of MRE samples with $0^{\circ}$, $45^{\circ}$, and $90^{\circ}$ mold orientation using the new configuration of magnetic flux mold enhanced 
the alignment of CIPs by providing more magnetic flux flows into the samples during the curing process. This consequently increased the MRE's maximum value of stiffness due to particles' strong interaction and providing a higher range modulus of MRE. In addition, the orientation of CIPs and their movement in MRE were much easier with the help of $15 \mathrm{wt} . \% \mathrm{SO}$ as a plasticizer and the CIPs were denoted closer to each other during the curing process, for all orientations. However, it can be seen from the experimental results that the MR effect of MRE maximizes its value for the orientation of using $45^{\circ}$ mold. The order of maximum modulus $\left(\mathrm{G}_{\max }\right)$ and MR effect of MRE versus orientations of CIPs are $45^{\circ}>0^{\circ}>90^{\circ}$, which is consistent with the findings of Boczkowa et al. [18] and Yao et al. [19]. Furthermore, the resultant MR effect of the MRE with $45^{\circ} \mathrm{CIPs}$ oriented is much larger due to the enhancement of the orientation of CIPs via new flux configuration mold during the pre-structure process of the material. The magneto-induced modulus and relative MR effects have reached 1.01 MPa and 918\%, respectively. Both have exceeded the results from Tian et al. [26] and Yao et al. [19] who depicted a value of $0.3 \mathrm{MPa}$ and $0.59 \mathrm{MPa}$ for the magneto-induced MR effect of MRE, respectively. Meanwhile, MRE with mold $0^{\circ}$ and $90^{\circ}$ to the $y$-axis and with isotropic distribution exhibit a lower value of relative MR effect which are $646 \%, 433 \%$, and $343 \%$, respectively.

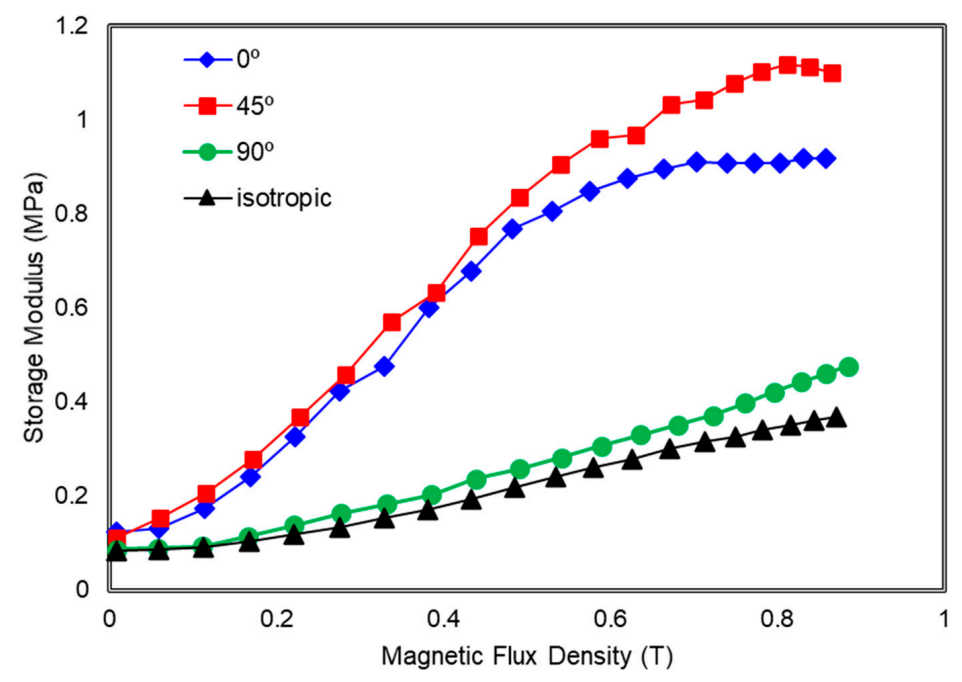

(a)

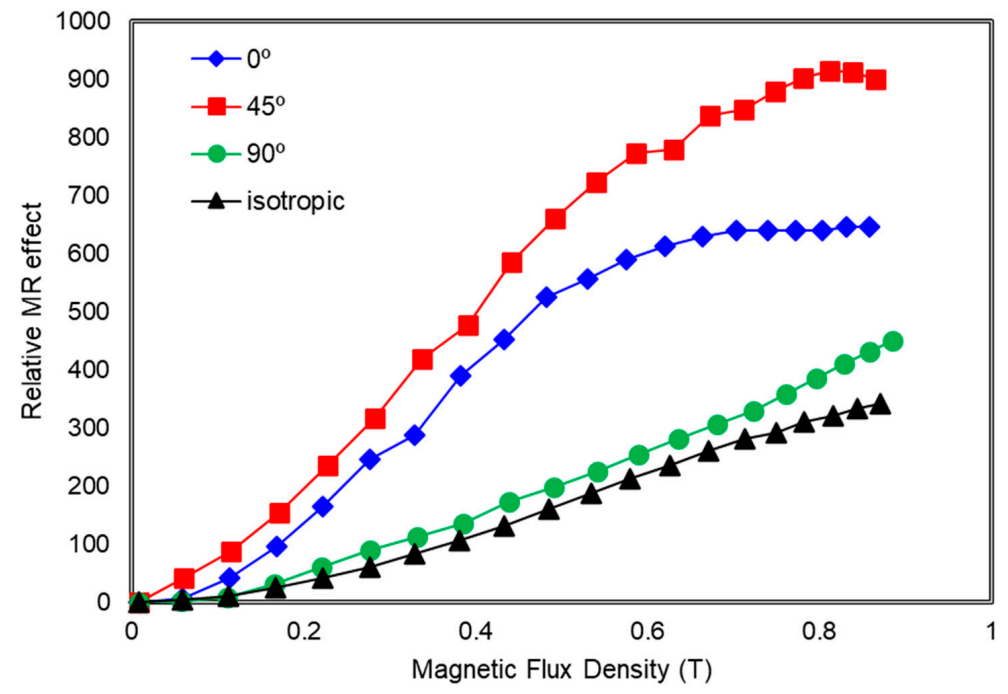

(b)

Figure 9. MRE with the various orientation mold of $0^{\circ}, 45^{\circ}, 90^{\circ}$ and isotropic (a) Storage modulus (b) Relative MR Effect as a function of magnetic swept. 
Table 3. The initial storage modulus $\left(\mathrm{G}_{0}\right)$, the magnetic-induce modulus $(\Delta \mathrm{G})$ and the MR effect of MRE samples with different mold orientations.

\begin{tabular}{ccccc}
\hline Sample & $\mathbf{G}_{\mathbf{0}}(\mathbf{M P a})$ & $\mathrm{G}_{\max }(\mathbf{M P a})$ & $\Delta \mathbf{G}(\mathbf{M P a})$ & Relative MR Effect (\%) \\
\hline $0^{\circ}$ & 0.12 & 0.92 & 0.79 & 646 \\
\hline $45^{\circ}$ & 0.11 & 1.12 & 1.01 & 918 \\
\hline $90^{\circ}$ & 0.09 & 0.48 & 0.39 & 433 \\
\hline isotropic & 0.08 & 0.37 & 0.29 & 343 \\
\hline
\end{tabular}

Under the influence of the magnetic field, the orientation or chains of CIPs tend to be set according to the direction of the magnetic field to minimize the Zeeman energy, which involves the displacement of the particles. Because the CIPs are restrained by interactions at the interface between the particle-polymer phase and embedded in the elastomeric matrix, their displacements were introduced by additional shear stress into the matrix, especially at the orientation of $45^{\circ}$, causing an increased in stress concentration of the matrix and between the CIPs, consequently increased the stiffness of the whole MRE [32]. This can be attributed to the fact that the resultant MR effect of MRE is much higher for the orientation of $45^{\circ}$, followed by $0^{\circ}$ and $90^{\circ}$ as shown in Figure $9 \mathrm{~b}$. It also is noted that the lowest magneto induced, $\Delta \mathrm{G}$ and relative MR effect have been obtained for MRE that has scattered CIPs (isotropic MRE).

Figure 10 shows the loss factor of MRE samples with various orientations of CIPs under different magnetic fields. As can be seen from the figure, particularly below $0.2 \mathrm{~T}$, all MRE samples with $0^{\circ}, 45^{\circ}$, and $90^{\circ}$ of mold orientations show a low value of loss factor with the isotropic MRE exhibited the lowest value, around 0.1 MPa. However, as the magnetic flux density increased up to $0.6 \mathrm{~T}$, MRE with $45^{\circ}$ orientations of mold has the highest loss factor which is more than $0.25 \mathrm{MPa}$ due to it giving the highest loss modulus compared to other orientations. Wang et al. [33] revealed that the loss factor or damping of the MRE is mostly caused by the interfacial friction between the magnetic particles and the rubber matrix.

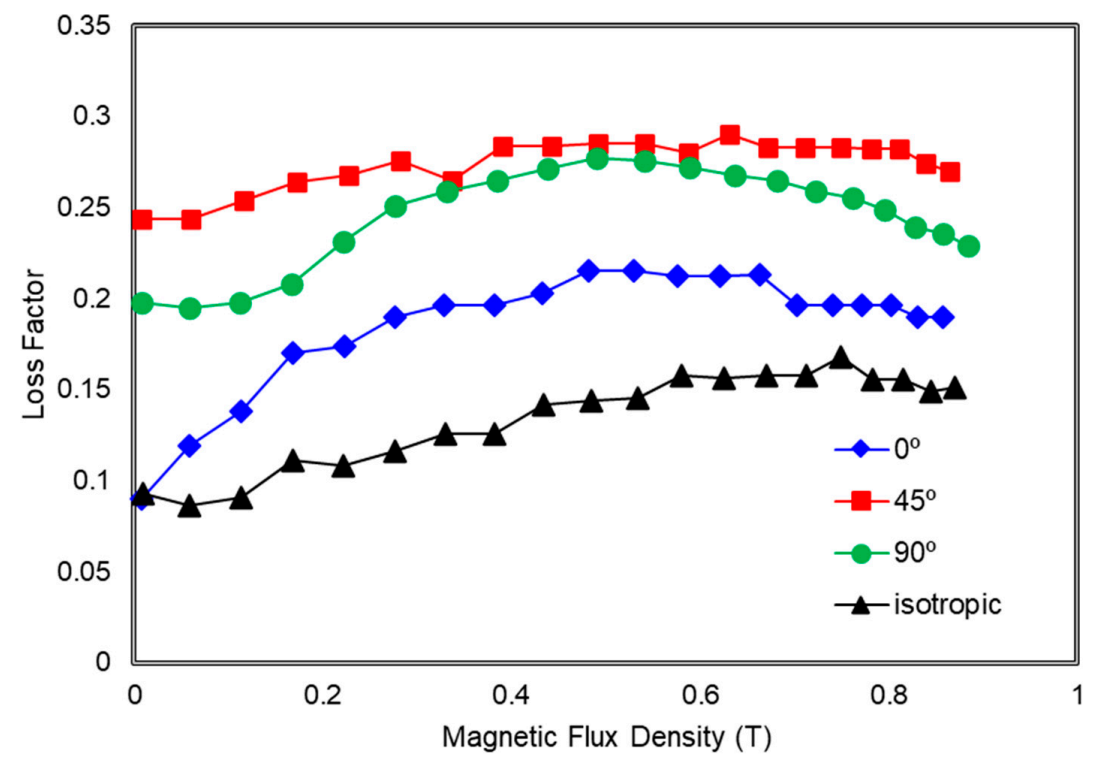

Figure 10. Loss Factor of MRE with the various orientation mold of $0^{\circ}, 45^{\circ}, 90^{\circ}$ and isotropic as a function of magnetic swept.

It is noted that the material that exhibits higher storage modulus would also possess higher loss modulus and higher loss factor. Therefore, rubber-based material such as MRE is normally used for damping material. For the current research, it can be observed that 
the interaction of matrix and particles is also relatively weak at $45^{\circ}$ orientation of mold thus, the sliding energy dissipation is produced easily under the applied shear stress. This phenomenon occurred when the magnetic particles are arranged at $45^{\circ}$ in the MRE and the shear force is in a horizontal direction; the force generated between the particles would increase the energy dissipation, which is caused by the interfacial sliding hence increasing the energy loss [33]. However, when the higher magnetic field is applied to the MRE sample regardless of the mold orientation, because of the strong interaction force between the particles and the rubber matrix, the sliding displacement can be decreased. As a result, energy dissipation and the loss factor are lowered. Furthermore, due to the interaction between the magnetic particles, the rubber matrix is more restricted at higher applied magnetic fields. As a result, the molecular rubber's energy dissipation is decreased.

\subsection{Rheological Properties: Effect of SO}

The effect of SO content on the storage and relative MR effect under different magnetic fields of MRE $45^{\circ}$ samples are shown in Figure 11. Meanwhile, the related initial storage modulus and MR effect data for each sample are summarized in Table 4.

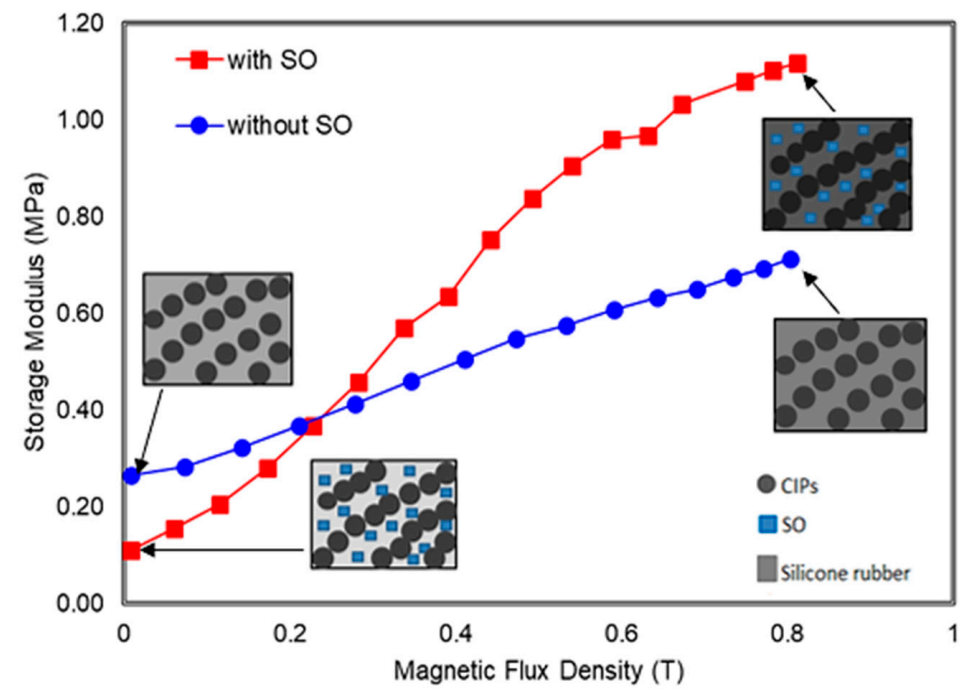

(a)

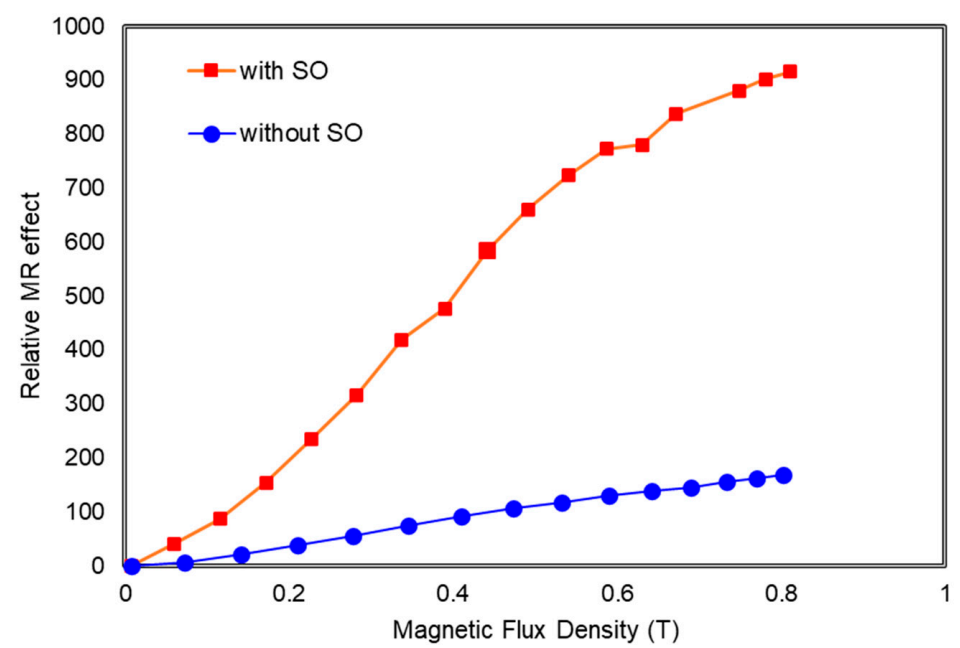

(b)

Figure 11. MRE with orientation mold of $45^{\circ}$ with and without SO (a) Storage modulus and (b) Relative MR effect as a function of magnetic swept. 
Table 4. The initial storage modulus $\left(\mathrm{G}_{0}\right)$, the magnetic-induce modulus $(\Delta \mathrm{G})$ and the MR effect of MRE $45^{\circ}$ with and without plasticizer.

\begin{tabular}{ccccc}
\hline Sample & $\mathbf{G}_{\mathbf{0}}(\mathbf{M P a})$ & $\mathrm{G}_{\max }(\mathbf{M P a})$ & $\Delta \mathrm{G}(\mathbf{M P a})$ & Relative MR Effect (\%) \\
\hline Without plasticizer & 0.26 & 0.71 & 0.45 & 173 \\
\hline With plasticizer & 0.11 & 1.12 & 1.01 & 918 \\
\hline
\end{tabular}

It can be observed that both magneto-induced $(\Delta G)$ and relative MR effect of the MRE $45^{\circ}$ sample increased with the magnetic field; however, the MRE effect increased greatly with the sample with $\mathrm{SO}$. The maximum storage modulus $\left(\mathrm{G}_{\max }\right)$ for the MRE with $\mathrm{SO}$ reaches the maximum state at $0.8 \mathrm{~T}$ as shown in Figure 11a that was presented with the schematic micrograph. This mechanism has demonstrated how the increased magnetic field has stiffened the MRE sample that originally had different initial storage modulus, $\mathrm{G}_{0}$ which depending whether it was with or without $\mathrm{SO}$. The grey intensity of the illustrated micrograph MRE becomes darker shade, showing the stiffer MRE as correspond to higher applied magnetic fields. In the presence of magnetic field, the addition of SO in the MRE $45^{\circ}$ would make it stiffer as compared to MRE $45^{\circ}$ without SO. Therefore, it can be briefly concluded that the magneto-induce storage modulus of the MRE $45^{\circ}$ with SO particularly $15 \mathrm{wt} . \%$ SO exhibits $1.01 \mathrm{MPa}$ with MR effect of $918 \%$, which is 5 times bigger than that of MRE $45^{\circ}$ without $\mathrm{SO}$ as in Figure $11 \mathrm{~b}$. This result exceeds the results from the literature with a depicted value of $0.6 \mathrm{MPa}$ for the magneto-induced MR effect of MRE $[19,25]$. The associated improvement on the relative and magneto-induced MR effect of the MRE $45^{\circ}$ by adding $S O$ was achieved using the new configuration of magnetic flux concept, which is believed enhancing the alignment of CIPs by providing more magnetic flux flows into the MRE $45^{\circ}$ which using the different approach as compared to Tian et al. [25] and Yao et al. [19]. Furthermore, the mobility and alignment of CIP's were much easier during the curing process, especially when the content of SO used was $15 \mathrm{wt} . \%$. This can be proven by determining the viscosity of the uncured MRE with and without SO as shown in Figure 12 under the different shear rates.

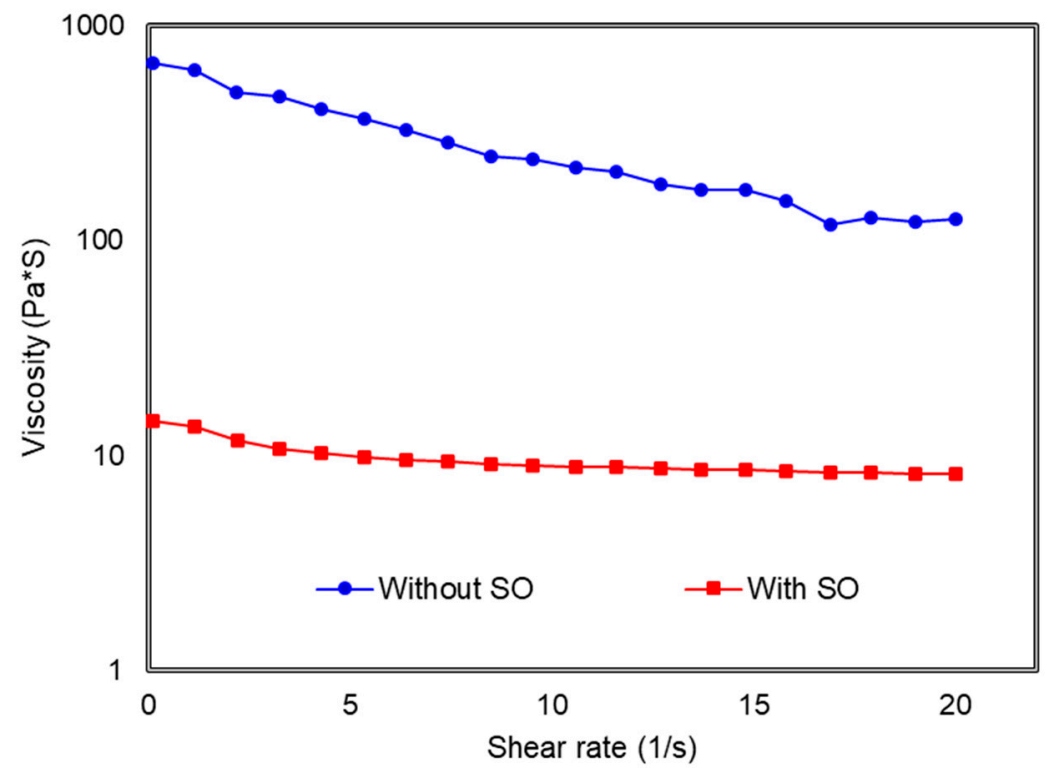

Figure 12. The viscosity of the uncured MRE samples with and without SO under the different shear rates.

The viscosity of the matrix phase during the preparation of the MRE melt would determine the elasticity of the cured MRE. The most important processes in the fabrication of the MRE are the dispersion and homogeneous distribution of the CIPs in the rubber 
matrix. It is also well known that the matrix's viscosity has a significant impact on the magnetic particles' dispersion [34]. For the MRE without SO, the CIPs are difficult to disperse, and consequently mobility of the CIPs is restricted during the curing process. Meanwhile, the viscosity of the melt MRE decreased with the addition of $15 \mathrm{wt} . \%$ of SO then helps CIPs to align easily following the applied magnetic flux during the curing process, which resulted in the enhancement of the rheological properties of the MRE $45^{\circ}$. Figure 12 also depicted the increment of shear rate has reduced the viscosity of both MRE $45^{\circ}$ samples. The molecular chain structures in the rubber matrix have experienced breakdowns upon applied shear stress and need time to recover hence reducing the crosslink density of the rubber that lowering the viscosity of the uncured MRE [35]. Associated with lower initial storage modulus and improved interaction between aligned CIPs with the addition of SO, the MR effect of MRE at $45^{\circ}$ orientation mold has been improved [19] upon using a new configuration magnetic flux mold.

\subsection{Rheological Properties: Effect of Strain Amplitude and Frequency}

The magnetic field-induced modulus changes of the MRE $45^{\circ}$ at three different strain amplitudes are shown in Figure 13. When the shear strain amplitude is $0.01 \%$ and $0.1 \%$, the initial field modulus of the sample is almost the same. However, as the strain amplitude increases to $1 \%$, the initial field modulus decreases from $0.38 \mathrm{MPa}$ to $0.34 \mathrm{MPa}$. This is due to the sliding effect between particles and matrix increased during the motion of the rubber molecular chains. Therefore, the interaction between molecular chains and particles with the matrix might be broken which leads to the decrement at the initial field modulus. The magneto-induced modulus of the MRE $45^{\circ}$ sample at strain amplitudes of $0.01 \%, 0.1 \%$ and $1 \%$ are $0.73,0.69$, and $0.46 \mathrm{MPa}$, respectively. The phenomenon that occurred is due to the increment of the distance among particles with the increase of strain amplitude. Therefore, it resulted in the decrement of the interactive force between particles hence lower the magneto-induced modulus. This finding agrees with findings from previous studies [21,36].

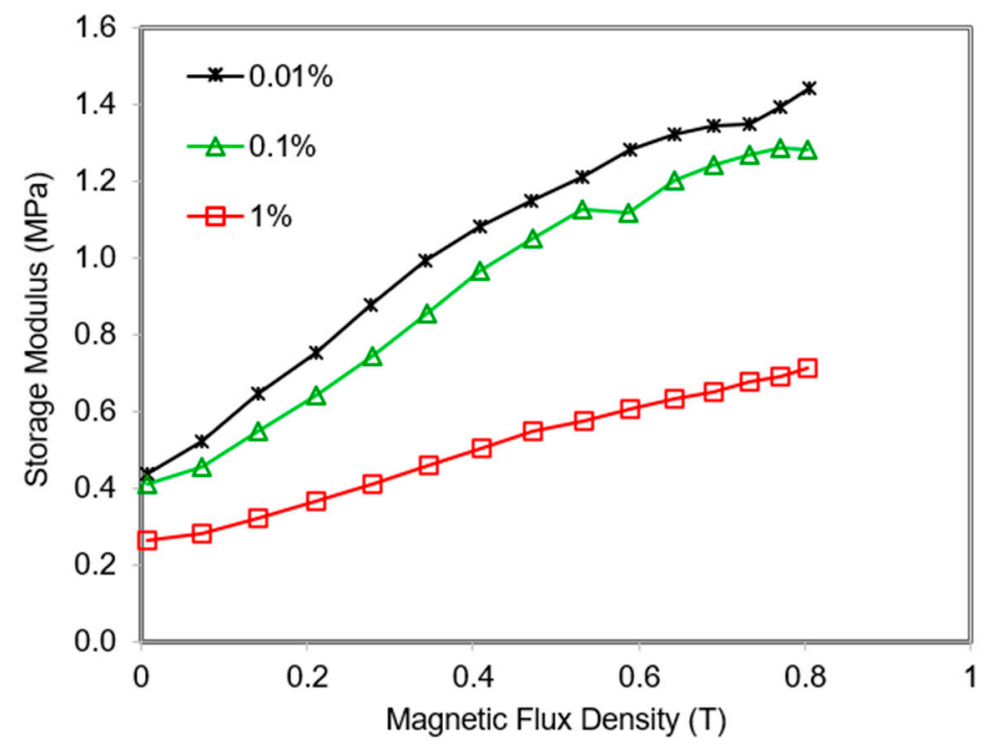

Figure 13. MRE $45^{\circ}$ at various amplitude strains as a function of magnetic swept.

On the other hand, the influence of the ramped magnetic flux on the shear storage modulus of MREs with different frequencies was evaluated. The motion of the polymer molecular chain is frequency-dependent, therefore the MR effect of MRE is also frequencydependent [21]. The MR effect of MRE $45^{\circ}$ at 1, 5, and $10 \mathrm{~Hz}$ is shown in Figure 14. It can be noted that the initial-field modulus increases with the increment of the test frequency. By increasing the frequency, the motion of MRE $45^{\circ}$ molecular chains cannot withstand the external stimuli, so molecular chains tend to be rigid and increased the 
value of the initial-field modulus. Meanwhile, all samples exhibited an increase in storage modulus as the intensity of the magnetic field increased. Attributed to the presence of a magnetic field, the attraction of the CIPs leads to the increased stiffness of MRE $45^{\circ}$ and simultaneously enhanced the corresponding storage modulus. Nevertheless, even though the storage modulus increased as the magnetic field increased, the initial field modulus also increased, resulting the magneto-induced modulus to be the same value of $1 \mathrm{MPa}$ although the samples were conducted at different frequencies. This value reveals that the magneto-induced modulus is independent of the test frequency; however, the relative MR effect decreases with increasing the test frequency due to the high value of the initial-field modulus.

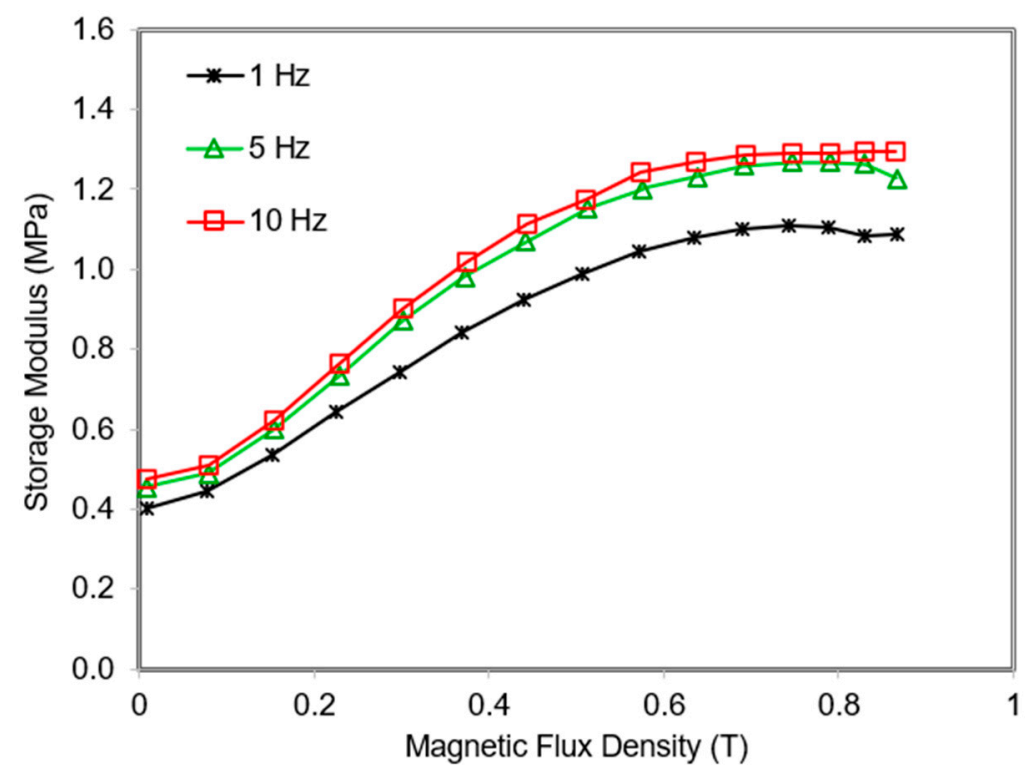

Figure 14. MRE $45^{\circ}$ at various frequencies as a function of magnetic swept.

\section{Conclusions}

In this study, MRE samples using an electromagnetic device with a new configuration mold with orientation of $0^{\circ}, 45^{\circ}$ and $90^{\circ}$ were successfully fabricated, and the overall properties, related to the morphological observation, magnetic and rheological properties were experimentally investigated. It has been shown from the morphological analysis that the CIPs are randomly dispersed for isotropic MRE and assembled into chain-like structures for anisotropic MRE. More specific results achieved from this work are summarized as follows.

(a) The new configuration of magnetic flux mold for curing device produces $0.315 \mathrm{~T}$ through simulation with uniform distribution of magnetic flux flow across the MRE sample.

(b) The magnetic mold was inserted in the magnetic coil without an air gap to evade magnetic flux losses. The magnetic molds have functioned as guidance for the concentrated magnetic flux to flow parallelly from top to bottom parts that passing through the MRE sample, which was sandwiched between the mold's parts for enhancing the alignment of CIPs in the MRE.

(c) The orientation of mold does alter the MR effect of MRE with the order of the storage modulus or MR effect versus orientations is $45^{\circ}>0^{\circ}>90^{\circ}$ with $45^{\circ}$ orientation achieved the highest relative MR effect of $909 \%$ and $1.01 \mathrm{MPa}$ for magneto-induced modulus. It is interesting to note that the finding exceeds the result from the literature that obtained $0.6 \mathrm{MPa}$ magneto-induced modulus based on $70 \mathrm{wt} . \% \mathrm{CIPs}$.

(d) Relative MR effect for MRE for mold orientation at $0^{\circ}, 90^{\circ}$ and isotropic distribution exhibited of $646 \%, 433 \%$ and $343 \%$, respectively.

(e) Plasticization is an effective method to improve the MR effect. Without the $\mathrm{SO}$, the relative MR effect obtained was only $173 \%$ with $0.45 \mathrm{MPa}$ magneto-induced modulus 
for MRE $45^{\circ}$. Meanwhile, the relative MR effect is dependent on the test frequency and strain.

The resultant MR effect of the MRE with $45^{\circ}$ mold oriented is much larger under shear deformation due to displacement of CIPs initiated additional shear stress into the matrix causing an increased in stress concentration of the matrix and between the CIPs, consequently increased the stiffness of the whole MRE. As a final note, the proposed MRE can be beneficial in industrial applications where they require a high range of stiffness, particularly under the shear deformation.

Author Contributions: Conceptualization, M.H.A.K. and S.A.M.; methodology, M.H.A.K., U. and N.N.; validation, M.H.A.K., U. and N.A.N.; writing—original draft preparation, M.H.A.K.; writingreview \& editing, M.H.A.K., S.A.M., N.A.N., S.A.A.A., U. and N.N. All authors have read and agreed to the published version of the manuscript.

Funding: This work was supported by the Ministry of Higher Education under Fundamental Research Grant Scheme (FRGS/1/2020/TKO/UTM/02/57), Japan International Cooperation Agency (Vot No. 4B696) and Professional Development Research University Grant (Vot No. 05E58). Authors also appreciate funding from Ristek-BRIN, Indonesia, through World Class Research Grant 2021.

Institutional Review Board Statement: Not applicable.

Informed Consent Statement: Not applicable.

Data Availability Statement: Not applicable.

Conflicts of Interest: The authors declare that there is no conflict of interest.

\section{References}

1. Hapipi, N.; Aziz, S.A.A.; Mazlan, S.A.; Ubaidillah; Choi, S.B.; Mohamad, N.; Khairi, M.H.A.; Fatah, A.Y.A. The field-dependent rheological properties of plate-like carbonyl iron particle-based magnetorheological elastomers. Results Phys. 2019, 12, $2146-2154$. [CrossRef]

2. Gan, R.; Li, Y.; Qi, S.; Zhu, M.; Yu, M. Study on the effect of particles size on viscoelastic properties of magnetorheological Elastomers. Curr. Smart Mater. 2019, 4, 59-67. [CrossRef]

3. Yunus, N.; Mazlan, S.; Ubaidillah; Abdul Aziz, S.; Tan Shilan, S.; Abdul Wahab, N. Thermal stability and rheological properties of epoxidized natural rubber-based magnetorheological Elastomer. Int. J. Mol. Sci. 2019, 20, 746. [CrossRef] [PubMed]

4. Bastola, A.; Hossain, M. Enhanced performance of core-shell hybrid magnetorheological elastomer with nanofillers. Mater. Lett. 2021, 297, 129944. [CrossRef]

5. Li, W.; Qi, S.; Zhu, M.; Xia, D.; Fu, J.; Yu, M. Improving transient magnetorheological response of magnetorheological elastomer by incorporating CIP@FeNi particles. Smart Mater. Struct. 2021, 30, 024002. [CrossRef]

6. Xia, L.; Hu, Z.; Sun, L. Micromechanics-based simulation of anisotropic magneto-mechanical properties of magnetorheological elastomers with chained microstructures. Smart Mater. Struct. 2021, 30, 2021. [CrossRef]

7. Khairi, M.H.A.; Mazlan, S.A.; Ubaidillah; Choi, S.; Aziz, S.A.A.; Mohamad, N.; Hapipi, N.M.; Nordin, N. Role of additives in enhancing the rheological properties of magnetorheological solids: A review. Adv. Eng. Mater. 2019, 21, 1800696. [CrossRef]

8. Samal, S.; Škodová, M.; Abate, O.; Blanco, I. Magneto-rheological elastomer composites. A review. Appl. Sci. 2017, 10, 124-134.

9. Vatandoost, H.; Norouzi, M.; Alehashem, S.M.S.; Smoukov, S.K. A novel phenomenological model for dynamic behavior of magnetorheological elastomers in tension-compression mode. Smart Mater. Struct. 2017, 26. [CrossRef]

10. Samal, S.; Škodová, M.; Blanco, I. Effects of filler distribution on magnetorheological silicon-based composites. Materials 2019, 12, 3017. [CrossRef]

11. An, J.S.; Kwon, S.H.; Choi, H.J.; Jung, J.H.; Kim, Y.G. Modified silane-coated carbonyl iron/natural rubber composite elastomer and its magnetorheological performance. Compos. Struct. 2017, 160, 1020-1026. [CrossRef]

12. Aziz, S.A.A.; Mazlan, S.A.; Ubaidillah, U.; Shabdin, M.K.; Yunus, N.A.; Nordin, N.A.; Choi, S.B.; Rosnan, R.M. Enhancement of viscoelastic and electrical properties of magnetorheological elastomers with nanosized Ni-Mg cobalt-ferrites as fillers. Materials $2019,12,3531$.

13. Zhao, D.; Cui, J.; Dai, X.; Liu, S.; Dong, L. Magneto-piezoresistive characteristics of graphene/room temperature vulcanized silicon rubber-silicon rubber magnetorheological elastomer. J. Appl. Polym. Sci. 2021, 138, 1-13. [CrossRef]

14. Liu, M.; Zhang, M.; Zhang, J.; Qiao, Y.; Zhai, P. Experimental investigation on the effect of graphene oxide additive on the steady-state and dynamic shear properties of PDMS-based magnetorheological elastomer. Polymers 2021, $13,1777$.

15. Gong, X.L.; Chen, L.; Li, J.F. Study of utilizable magnetorheological elastomers. Int. J. Mod. Physic B 2007, $21,4875-4882$. [CrossRef] 
16. Lokander, M.; Stenberg, B. Improving the magnetorheological effect in isotropic magnetorheological rubber materials. Polym. Test. 2003, 22, 677-680. [CrossRef]

17. Chen, L.; Gong, X.L.; Jiang, W.Q.; Yao, J.J.; Deng, H.X.; Li, W.H. Investigation on magnetorheological elastomers based on natural rubber. J. Mater. Sci. 2007, 42, 5483-5489. [CrossRef]

18. Boczkowska, A.; Awietjan, S.F.; Pietrzko, S.; Kurzydłowski, K.J. Mechanical properties of magnetorheological elastomers under shear deformation. Compos. Part B Eng. 2012, 43, 636-640. [CrossRef]

19. Yao, J.; Yang, W.; Gao, Y.; Scarpa, F.; Li, Y. Magnetorheological elastomers with particle chain orientation: Modelling and experiments. Smart Mater. Struct. 2019, 28, 095008. [CrossRef]

20. Kimura, Y.; Kanauchi, S.; Kawai, M.; Mitsumata, T.; Tamesue, S.; Yamauchi, T. Effect of plasticizer on the magnetoelastic behavior for magnetic polyurethane elastomers. Chem. Lett. 2015, 44, 177-178. [CrossRef]

21. Wu, J.; Gong, X.; Fan, Y.; Xia, H. Improving the magnetorheological properties of polyurethane magnetorheological elastomer through plasticization. J. Appl. Polym. Sci. 2012, 123, 2476-2484. [CrossRef]

22. Immergut, E.H.; Mark, H.F. Principles of Plasticization. In Plasticization and Plasticizer Processes; ACS Publications: Washington, DC, USA, 1965; Volume 48, pp. 1-26. ISBN 9780841200494.

23. Awagon, A. Introduction to Rubber Elasticity; Society of Rubber Science and Technology: Tokyo, Japan, 1996; Volume 69, ISBN 1859571506.

24. Kallio, M.; Lindroos, T.; Aalto, S.; Järvinen, E.; Kärnä, T.; Meinander, T. Dynamic compression testing of a tunable spring element consisting of a magnetorheological elastomer. Smart Mater. Struct. 2007, 16, 506-514. [CrossRef]

25. Tian, T.; Nakano, M. Fabrication and characterisation of anisotropic magnetorheological elastomer with $45^{\circ}$ iron particle alignment at various silicone oil concentrations. J. Intell. Mater. Syst. Struct. 2018, 29, 151-159. [CrossRef]

26. Li, Y.; Li, J.; Li, W.; Du, H. A state-of-the-art review on magnetorheological elastomer devices. Smart Mater. Struct. 2014, 23. [CrossRef]

27. Samal, S.; Kolinova, M.; Blanco, I. The magneto-mechanical behavior of active components in iron-elastomer composite. J. Compos. Sci. 2018, 2, 54. [CrossRef]

28. Nayak, B.; Dwivedy, S.K.; Murthy, K.S.R.K. Fabrication and characterization of magnetorheological elastomer with carbon black. J. Intell. Mater. Syst. Struct. 2015, 26, 830-839. [CrossRef]

29. Suo, S.; Xu, Z.; Xu, F. A model of magnetorheological elastomer based on chi-square distribution. J. Funct. Mater. 2016, 47, 9063-9067.

30. Davis, L.C. Model of magnetorheological elastomers. J. Appl. Phys. 1999, 85, 3348-3351. [CrossRef]

31. Samal, S.; Kolinova, M.; Blanco, I.; Poggetto, G.D.; Catauro, M. Magnetorheological Elastomer Composites: The Influence of Iron Particle Distribution on the Surface Morphology. Macromol. Symp. 2020, 389, 2-5. [CrossRef]

32. Szymczyk, W.; Boczkowska, A.; Niezgoda, T.; Zubko, K. Experimental Validation of Numerical Methods of MRE Simulations. Solid State Phenom. 2009, 154, 113-120. [CrossRef]

33. Wang, Y.; Zhang, X.; Oh, J.; Chung, K. Fabrication and properties of magnetorheological elastomers based on CR/ENR selfcrosslinking blends. Smart Mater. Struct. 2015, 24, 95006. [CrossRef]

34. Ge, L.; Gong, X.L.; Fan, Y.; Xuan, S. Preparation and mechanical properties of the magnetorheological elastomer based on natural rubber/rosin glycerin hybrid matrix. Smart Mater. Struct. 2013, 22, 115029. [CrossRef]

35. Fan, Y.; Gong, X.L.; Xuan, S.; Qin, L.; Li, X. Effect of cross-link density of the matrix on the damping properties of magnetorheological elastomers. Ind. Eng. Chem. Res. 2013, 52, 771-778. [CrossRef]

36. Ubaidillah; Imaduddin, F.; Li, Y.; Mazlan, S.A.; Sutrisno, J.; Koga, T.; Yahya, I.; Choi, S.B. A new class of magnetorheological elastomers based on waste tire rubber and the characterization of their properties. Smart Mater. Struct. 2016, 25, 1-15. [CrossRef] 